\title{
1 Decoding and perturbing decision states in real time
}

2 Diogo Peixoto ${ }^{1,2 *}$, Jessica R. Verhein ${ }^{3,4}$, Roozbeh Kiani, Jonathan C. Kao ${ }^{6,7,8}$, Paul

3 Nuyujukian $3,6,9,10,15,16$, Chandramouli Chandrasekaran $6,11,12,13$, Julian Brown ${ }^{11}$, Sania Fong ${ }^{11}$,

4 Stephen I. Ryu ${ }^{6,14}$, Krishna V. Shenoy ${ }^{1,6,9,11,15,16}$, William T. Newsome $e^{1,11,15,16}$

51 Neurobiology Department, Stanford University, Stanford, CA 94305

62 Champalimaud Neuroscience Programme, Lisbon, Portugal, 1400-038

73 Neurosciences Graduate Program, Stanford University, Stanford, CA 94305

84 Medical Scientist Training Program, Stanford University School of Medicine, Stanford, CA

994305

105 Center for Neural Science, New York University, New York, NY 10003

116 Electrical Engineering Department, Stanford University, Stanford, CA 94305

127 Department of Electrical and Computer Engineering, University of California, Los Angeles, CA

1390095

148 Neurosciences Program, University of California, Los Angeles, CA 90095

159 Bioengineering Department, Stanford University, Stanford, CA 94305

1610 Neurosurgery Department, Stanford University, Stanford, CA 94305

1711 Howard Hughes Medical Institute, Stanford University, CA 94305

1812 Department of Psychological and Brain Sciences, Boston University, Boston, MA 02215

1913 Department of Anatomy and Neurobiology, Boston University School of Medicine, Boston,

$20 \quad$ MA 02118

2114 Neurosurgery Department, Palo Alto Medical Foundation, Palo Alto, CA 94301

$2215 \mathrm{Wu}$ Tsai Neurosciences Institute, Stanford University, Stanford, CA 94305 
2316 Bio-X Institute, Stanford University, Stanford, CA 94305

$24 *$ Denotes equal contribution.

25 Please address correspondence to Bill Newsome (bnewsome@stanford.edu), Diogo Peixoto

26 (dpeixoto@stanford.edu) or Jessica Verhein (jverhein@stanford.edu).

\section{Summary}

29 In dynamic environments, subjects often integrate multiple samples of a signal and combine them

30 to reach a categorical judgment. The process of deliberation on the evidence can be described by

31 a time-varying decision variable (DV), decoded from neural activity, that predicts a subject's

32 decision at the end of a trial. However, within trials, large moment-to-moment fluctuations of the

33 DV are observed. The behavioral significance of these fluctuations and their role in the decision

34 process remain unclear. Here we show that within-trial DV fluctuations decoded in real time from

35 motor cortex are tightly linked to choice behavior, and that robust changes in DV sign have the

36 statistical regularities expected from behavioral studies of changes-of-mind. Furthermore, we find

37 single-trial evidence for absorbing decision bounds. As the DV builds up, heavily favoring one or

38 the other choice, moment-to-moment variability in the DV is reduced, and both neural DV and

39 behavioral decisions become resistant to additional pulses of sensory evidence as predicted by

40 diffusion-to-bound and attractor models of the decision process. 
44 When making a categorical decision about a noisy stimulus, it is common to fluctuate between

45 levels of commitment to a choice before reporting a decision. In some instances the fluctuations

46 are sufficiently strong to lead to a "change of mind" (CoM) while deliberating ${ }^{1-6}$ or even while the

47 reporting action is being executed ${ }^{7}$. Because these within-trial fluctuations are different from trial

48 to trial and not necessarily tied to an external event or stimulus feature, they can only be captured

49 using a moment-to-moment neural readout of the decision state on single trials.

50 To obtain this readout, we decoded a decision variable (DV) from neural population activity in

51 PMd and M1 in real time to continuously estimate the decision state while two monkeys performed

52 a motion discrimination $\operatorname{task}^{8,9}$ (Fig. 1a, see Methods). The DV was estimated by applying a linear

53 decoder, trained on data from a previous experimental session, to spiking data (from 96 to 192

54 electrodes) from the preceding $50 \mathrm{~ms}$, updated every $10 \mathrm{~ms}$ throughout each trial (Fig. 1b, see

55 Methods). The sign of the DV indicated which choice was predicted by the decoder, which allowed

56 us to calculate the decoder's prediction accuracy. The DV magnitude reflected the confidence of

57 the model's prediction in units of log-odds for one vs. the other decision (see Methods). Note that

58 the decision variable as defined here encompasses all choice predictive signals that can be decoded

59 from neural activity ${ }^{10}$, including but not limited to moment-to-moment value of accumulated

60 evidence as posited in classical sequential sampling models.

61 We have previously demonstrated with offline analysis that this decision variable (DV) can predict

62 choices on single trials up to seconds before initiation of the operant response, and that the

63 accuracy of these predictions increases on average throughout the course of the trial ${ }^{10}$.

64 Here, we employed closed-loop, neurally-contingent control over stimulus timing to directly probe

65 the relationship of within-trial DV fluctuations to behaviorally meaningful decision states. For the 
66 first time, we quantified the behavioral effects of previously covert DV variations (i) as a function

67 of time and for different virtual DV boundaries imposed during the trial, (ii) when large, CoM-like

68 fluctuations were detected during deliberation on noisy visual evidence, and (iii) when

69 subthreshold stimulus pulses were added during the trial.

70 Having a nearly instantaneous real-time estimate of the decision state read-out enabled us to

71 terminate the visual stimulus based on the current value (or history) of the DV and validate the

72 behavioral relevance of DV fluctuations using the monkey's behavioral reports following stimulus

73 termination.

74 Decisions on perceived stimulus motion can be reliably decoded in real time based on $50 \mathrm{~ms}$ of PMd/M1 neural activity

77 Two monkeys performed a variable duration variant of the classical random dot motion

78 discrimination task using an arm movement as the operant response ${ }^{10}$. As expected, the subjects

79 performed better for higher coherence and longer duration stimuli and reached almost perfect

80 performance for the easiest stimuli (Extended Data Fig. 1).

81 We first measured the accuracy of our real-time decoder in predicting the monkeys' behavioral

82 choices as a function of time during the trial. As in our previous offline results ${ }^{10}$, average prediction

83 accuracy started at chance levels during the targets epoch (Fig. 1c, Extended Data Fig. 2a). During

84 the dots presentation average prediction accuracy quickly departed from baseline (174.5 $\mathrm{ms} \pm 18.8$

85 and $214.5 \mathrm{~ms} \pm 8.09 \mathrm{~ms}$ after dots onset for monkey $\mathrm{H}$ and $\mathrm{F}$, respectively), rising monotonically

86 for the rest of the epoch. The rise in prediction accuracy was steep, reaching 99\% (98\%) correct

87 for the longest stimuli presentations for monkey $\mathrm{H}(\mathrm{F})$, respectively. Moreover, for all 4 epochs 
considered (targets, dots, delay and post-go) the average accuracy difference between our real-

89 time readout and the equivalent one calculated offline (trained using data from the same session)

90 was within $\mathrm{a} \pm 2 \%$ range (Extended Data Fig. 3a-d). Thus, our real-time choice decoder reproduces

91 prediction accuracy as reported in previous off-line analyses of decision-related neural activity in

92 both the oculomotor and somatomotor systems ${ }^{1,10}$.

93 Our real-time decoder also reproduced the temporal dynamics and coherence dependence of the

94 DV, as reported in previous off-line studies ${ }^{1,10}$. The on-line DV: (i) started around 0 at the time of

95 dots onset, (ii) separated by choice after $200 \mathrm{~ms}$, and (iii) rose (or fell) faster for easier trials (Fig.

96 1d, Extended Data Fig. 2b; regression of DV onto coherence significant for both choices, $\mathrm{p}<10^{-5}$

97 uncorrected). Prediction accuracy was higher for correct trials compared to error trials (Extended

98 Data Fig. 4) when holding the stimulus coherence constant, as expected from previous studies ${ }^{11}$.

99 Finally, our decoding method yielded stable performance across multiple days, justifying

100 combination of data across sessions (Extended Data Fig. 5). This is particularly important when

101 studying rare events such as CoMs, which only happen on a small fraction of the trials and could

102 not be characterized adequately using a single session's data.

103 Real time DV closely predicts choice likelihood across experimental conditions

104 The previous results are a proof of concept for a highly reliable, real-time readout of decision state

105 in PMd/M1 using spiking data from 100-200 units and aggregate and average metrics (Fig. 1c-

106 d, Extended Data Fig. 2a-b). However, we often observed large fluctuations (over 3 natural log

107 units) in the decision variable on individual trials, even within single behavioral epochs (Fig. 1e).

108 If moment-to-moment fluctuations in DV during single trials (as estimated by our decoder) reflect

109 true fluctuations in the decision state of the animal, we expect larger absolute values of DV to be 
110 associated with stronger preference for one of the two choices, and hence higher prediction

111 accuracy were a decision to be required at any time during a single trial.

112 Because we decoded and tracked the DV in real-time, we were able to terminate the visual stimulus

113 in a neurally contingent manner and probe both neural activity and the subject's behavior with

114 high precision and negligible latency ( $<34 \mathrm{~ms}$, see Methods). Inspired by sequential sampling

115 behavioral models that assume a bound ${ }^{12-14}$, the first closed-loop test we performed was to impose

116 virtual decision boundaries that, if reached, would result in stimulus termination (Fig. 2a),

117 prompting the subject to immediately report its decision (in trials with no delay period). In this

118 manner we obtained a direct mapping between the nearly instantaneous readout of decision state

119 and the likelihood of a given behavioral choice.

120 Figure $2 \mathrm{~b}$ shows 22 example DV traces from trials that led to stimulus termination by reaching a

121 fixed DV boundary of magnitude 3, within a tolerance of $\pm 0.25 \mathrm{DV}$ units.

122 To characterize the relationship between the DV at termination and prediction accuracy, we

123 systematically swept the parameter space for the boundary height using values spanning 0.5-5 DV

124 units in 0.5 increments (1DV unit corresponds to an increase of 2.718 in the likelihood ratio of

125 choosing one target over the other). Figure 2c shows that prediction accuracy increases

126 monotonically with the DV magnitude at termination as expected. Moreover, using only $100 \mathrm{~ms}$

127 of data to estimate the DV that triggered termination, the difference between the observed

128 likelihood of a given choice (solid trace) and that predicted by the logistic function (dashed trace)

129 was only, on average, 1.7\% (1.9\%) for monkey H (F) (Fig. 2c, Extended Data Fig. 2c). For

130 example, neural DV values of \pm 3 predict decisions upon termination with an accuracy of $98 \%$.

131 Even DV values as low as $\pm 0.5-1$ predict decisions with an accuracy of nearly $70 \%$. DV 
132 fluctuations below \pm 0.5 are more susceptible to noise in our estimates of decision state and at most

133 are associated with very weak choice preferences and were thus not tested. Overall, these results

134 show that moment-by-moment fluctuations in PMd/M1 neural population activity captured by our

135 decoding model are indeed reflective of a fluctuating internal decision state of the animal-

136 fluctuations that have been covert and thus uninterpretable until now.

137 Figure 2c (Extended Data Fig. 2c) combines trials across a wide range of coherences and stimulus

138 durations, aggregated across 17 (15) sessions from monkey $\mathrm{H}(\mathrm{F})$. To identify experimental factors

139 that might influence the observed relationship between DV at termination and prediction accuracy,

140 we first resorted the same trials in Figure $3 \mathrm{c}$ by stimulus coherence. The results show that there is

141 a small separation between the curves for high and low coherence trials (Fig. 2d) with higher

142 accuracy for high coherence trials. The shift is small but reliable across monkeys (Extended Data

143 Fig. 2d). We hypothesized that this difference resulted from motion energy signals already en route

144 from the retina to PMd/M1 ( $\sim 175 \mathrm{~ms}$ latency) when the DV reached stimulus termination. More

145 motion energy signals would be arriving from this neural 'pipeline' on high coherence trials,

146 leading to a slightly higher DV than we measured at stimulus termination.

147 To assess this possibility, we measured the derivative of the DV around termination and performed

148 the following two analyses. First we checked whether DV derivative explained a significant

149 fraction of choice variance beyond DV value alone (see Methods). For both monkeys the effect of

150 DV derivative (defined as the DV slope in the last $50 \mathrm{~ms}$ of stimulus presentation) was significant

$151\left(\mathrm{p}=0.02, \mathrm{p}=4.5 \times 10^{-11}\right.$ for monkey $\mathrm{H}$ and $\mathrm{F}$, respectively) and the effect was congruent with our

152 hypothesis: stronger positive derivatives predicted higher likelihood of rightward choices and

153 stronger negative derivatives predicted higher likelihood of leftward choices (Extended Data Table

154 1, "DV diff"). Second, we tested whether high coherence trials were associated with higher DV 
155 derivatives at termination by performing linear regression of DV derivatives as a function of signed

156 coherence. For both monkeys signed coherence was strongly predictive of DV slopes: $p=2.17$

$157 \times 10^{-171}$ and $\mathrm{R}^{2}=0.23$ for monkey $\mathrm{H}$ and $\mathrm{p}=1.57 \times 10^{-105}$ and $\mathrm{R}^{2}=0.16$ for monkey $\mathrm{F}$. These results

158 confirm that DV derivative is predictive of choice beyond DV alone and show that higher

159 coherence trials are associated with higher DV derivatives. The data are consistent with our

160 hypothesis above that the DV continues to evolve under the influence of 'pipeline' sensory

161 information for a short interval following stimulus termination, resulting in somewhat better

162 prediction accuracy than expected from the DV at termination, especially at high coherences.

163 Sorting trials by duration (Fig. 2e, Extended Data Fig. 2e), reveals a different effect: the centers of

164 the quantiles are strongly shifted to the right (higher DV magnitudes) for longer stimuli compared

165 to shorter stimuli. This effect is expected from multiple sequential sampling models ${ }^{8,15-17}$. In drift

166 diffusion models, for example, diffusion to high decision bounds requires more time than for low

167 bounds ${ }^{18}$. However, we tested whether stimulus duration per se was a significant predictor of

168 choice independently of DV value by including two additional regressors in our logistic model of

169 choice: stimulus duration (representing choice bias as a function of time) and an interaction term

170 between stimulus duration and direction (representing increased sensitivity to stimulus coherence

171 as function of time). Neither regressor was significant for either monkey ( $\mathrm{p}>0.05$, Extended Data

172 Table 1), implying that the likelihood of making one or the other choice depended on DV value

173 independently of the time required to reach that value.

174 Together, these results show that fluctuations in DV magnitude at a $100 \mathrm{~ms}$ time scale have a

175 predictable correlate in choice likelihood that is lawfully influenced by stimulus coherence and

176 robust across time. We emphasize that our decoded DV is model-based and thus a proxy for the

177 actual decision state in the brain. We are sampling from a relatively small number of neurons, and 
178 the underlying mechanism is unlikely to be strictly linear (in contrast to the logistic model). In

179 addition, we do not know with certainty when the deliberation process ends within the brain, which

180 could occur before or after our stimulus termination on individual trials. Despite these caveats, our

181 ability to predict choice likelihood using a DV boundary criterion at stimulus termination within a

182 very small margin of error $(<2 \%$ on average) confirms that DV is a reliable proxy for decision

183 state.

184 Neurally detected CoMs can be validated and match the statistical regularities expected from 185 previous studies

186 The mapping between DV and choice likelihood obtained in the first experiment (Fig. 2c), enabled

187 us to perform a new closed-loop experiment aimed at capturing particularly robust DV fluctuations

188 in which the sign of the DV (and thus the neurally inferred decision state of the animal) changed

189 in the middle of a trial, suggestive of a 'change of mind' at the behavioral level (CoM, Fig. 3a-b).

190 When the neural criteria for a CoM were met in real-time (see Methods, examples in Figure 4a,

191 orange and green arrows), the stimulus was terminated instructing the monkey to make a decision

192 as described above. Our aim was to detect neurally-based candidate CoMs, assess the influence

193 of the decision states before and after the CoM on the final choice, and determine whether

194 statistical properties of the neurally derived CoMs match the properties expected of CoMs from

195 prior psychophysical and neurophysiological studies.

196 We conceptually divide a CoM trial into two segments - the initial preference prior to the DV sign

197 change, and the final (opposite) preference that leads to the observed choice. The observed choices

198 allow corroboration of the neural estimate of the final decision state in the second segment

199 (Extended Data Fig. 6). For monkey F, the relationship between choice prediction accuracy and 
DV at stimulus termination for CoM trials was very similar to that of non-CoM trials (compare

201 Extended Data Fig. 2c and Extended Data Fig. 6b, mean error between predicted and observed

202 choice likelihood: $1.9 \%$ for non-CoM trials vs 3.8\% for CoM trials). This relationship was lawful

203 and monotonic for monkey $\mathrm{H}$ as well although lower than expected (Extended Data Fig. 6a,

204 compared to Fig. 2c, mean error between predicted and observed choice likelihood: 1.7\% for non-

205 CoM trials vs $9.3 \%$ for CoM trials), suggesting that in addition to the measured DV at stimulus

206 termination, monkey H's decisions were also influenced by some aspect of the DV trajectory

207 history specifically related to the CoM. We formally tested this hypothesis by regressing choice as

208 a function of 3 additional parameters (in addition to DV at termination) that were enforced and

209 monitored in this experiment (see Methods): maximum DV deflection before sign change and

210 duration of sign stability before and after DV sign change. For monkey F, no additional factor was

211 choice predictive, whereas for monkey $\mathrm{H}$ both the duration of sign stability before and after the

212 CoM were also choice predictive (Extended Data Table 2) as suspected from Extended Data Fig.

2136.

214 We combined all 985 (1727) CoM's detected in monkey H (F) to assess whether our neurally

215 detected CoMs conformed to three statistical regularities of CoMs established in previous

216 psychophysical $^{7}$ and electrophysiological ${ }^{1}$ studies.

217 The first observation is that CoMs are more frequent for low and intermediate coherence trials as

218 opposed to high coherence trials, as high coherences are more likely to lead to straightforward

219 integration of evidence toward the correct choice. We found the same to be true in our real-time

220 detection data (Fig. 3c, Extended Data Fig. 2f; linear regression p<0.001). 
221 The second observation is that CoMs are more likely to be corrective than erroneous. This

222 prediction results from the corrective role of additional visual evidence on the initial preference of

223 the subjects. This trend was also verified in the CoMs we detected with CoMs for non-zero

224 coherences and for both monkeys being more likely corrective than erroneous (Fig. 3d, Extended

225 Data Fig. 2g; Wilcoxon rank sum test $\mathrm{p}<0.001$, median corrective and erroneous CoM counts: 530

226 and 242 for monkey $\mathrm{H}$ and 1046 and 443 for monkey F, respectively).

227

228 Finally, the third observation made in these previous studies was that CoMs were more frequent

229 early in the trial than later in the trial, consistent with drift diffusion models in which the DV is

230 more likely to have hit an absorbing decision bound as the trial progresses. We observed this effect

231 in our real-time, neurally detected CoMs as well (Fig. 3e, Extended Data Fig. 2h).

232 We also discovered a new regularity associated with CoMs: the average time of zero crossing was

233 negatively correlated with stimulus coherence (Fig. 3f, Extended Data Fig. 2i). This observation

234 likely results from the stronger corrective effect of higher coherence stimuli (Fig. 3d, Extended

235 Data Fig. 2g).

236 Together, these results show that robust fluctuations in DV that imply a change in choice

237 preference (zero crossing) can be captured in real time and validated as changes of mind.

238 Pulses of additional visual motion evidence have smaller neural and behavioral effects when

239 presented at larger DV values

240 In a final set of closed-loop experiments whether the neural and behavioral responses to brief

241 pulses of additional motion information varied with the state of the DV before the pulse. Inspired

242 by decision-making models involving buildup of neural activity to a bound ${ }^{15,16,19,20}$, we expected 
243 termination of the deliberation process and commitment to a choice to be more likely at high DV

244 values ${ }^{1,7,8,16,21}$. We therefore hypothesized that additional pulses of sensory evidence would result

245 in less change in DV and behavior when pulses were triggered by high DV values.

247 To characterize the relationship between DV and responses to a stimulus pulse, we again imposed

248 virtual DV boundaries (as in Fig. 3a-b) that, if reached, triggered a 200-ms pulse of additive dots

249 coherence (randomly assigned to be rightward or leftward on each trial) followed by stimulus

250 termination (Fig. 4a). We swept a subset of the previously used DV values for the boundary

251 (spanning 1-4 DV units, in 1.0 increments). Pulse strength was calibrated to yield very small but

252 significant effects on behavior, in an effort to avoid making the pulses so salient as to change the

253 animals' integration strategy on pulse trials $(\Delta$ coherence $=2 \%$ for monkey $\mathrm{H}, 4.5 \%$ for monkey

254 F). Pulse information had no bearing on the reward ${ }^{8,17}$. Motion pulses slightly but significantly

255 biased the monkeys' choices in the direction of the pulse ( $p=8.38 \mathrm{E}-14$ for monkey H, Fig. 4b; $p$

$256=1.95 \mathrm{E}-4$ for monkey F, Extended Data Fig. 2j).

258 We reasoned that, to detect the presumably small effects of these small motion pulses on the DV,

259 we would need to account for a processing delay for changing stimulus information to influence

260 our recorded neural populations. Thus, to quantify the effect of the pulse on the evolving DV, we

261 first measured the minimum latency for visual stimulus information to influence the DV: we

262 calculated the time after stimulus onset at which the DV traces diverged for rightward vs. leftward

263 choices in an independent set of open loop trials at the strongest motion coherence. We refer to

264 this time point as the evidence representation latency (ERL). For each trial, we measured the

265 change in DV $(\Delta \mathrm{DV})$ for each time bin, beginning at the time of pulse onset plus the ERL (or 
PERL — see Methods). We found that, on average, motion pulses slightly but significantly biased $\Delta \mathrm{DV}$ in the direction of the pulse (Fig. 4c, Extended Data Fig. 2k).

In the case of simple, unbounded linear integration, we expect the magnitude of DV change in response to a fixed motion pulse to remain constant regardless of the triggering DV at pulse onset. when triggered by low as compared to high DV values.

274 Previous studies have shown that behavioral and LIP neural responses to similar motion pulses tend to be smaller when pulses are delivered later in the stimulus ${ }^{8,17}$. Large DV values tend to occur later in the trial, and this was hypothesized to be the underlying reason for the diminishing

277 pulse effects (assuming some sort of bound on integration of evidence at larger DVs); but these 278 studies lacked concurrent neural population recording and decoding and thus did not have access 279 to the momentary decision state. Thus, the time of pulse onset is a possible confound for the 280 decreasing pulse effects at high DV bound values as depicted in Fig 4d. To control for this 281 possibility, we first used the slope of the $\Delta \mathrm{DV}$ vs. time relationship measured on individual trials $282(\Delta \mathrm{DV}$ slope) to summarize the effect of the stimulus pulse on DV on single trials. We then 283 performed a multiple regression analysis of $\triangle \mathrm{DV}$ slope that included both the triggering $\mathrm{DV}$ value 284 and the time of the motion pulse as regressors (and other variables as well - see Methods). The 285 regression data confirm that the effect of stimulus pulses on DV is only significant when triggered 286 by lower DV values, and that this effect is not explained by pulse timing (Fig. 4e, Extended Data 287 Fig. 2m, Supplementary Information Table 1). Similarly, the effect of motion pulses on 288 psychophysical behavior is weaker when triggered by high DV values, and these effects also are 

2).

292 Our finding that larger DVs (and corresponding behavioral readouts) are more resistant to pulses 293 is consistent with several models of decision formation, including linear integration to a decision 294 bound (such as a simple stopping criterion ${ }^{12}$ ) or a more complex nonlinear integration 295 process $^{17,22,23}$. Inspired by these results, we returned to the data from the first two experiments in 296 an attempt to explore the nature of the apparent bounding mechanism by analyzing the time297 variance of the DV over the course of individual trials. In the case of an absorbing decision bound 298 or attractor network, we would expect DV variability (measured as the DV time derivative) to 299 decrease after reaching the bound. We indeed found that, on average, DV variability decreases 300 over time within single trials (Fig. 5a, Extended Data Fig. 7a). This effect holds across all stimulus 301 strengths, although variability peaks earlier and falls faster on the easiest trials (Fig. 5b, Extended 302 Data Fig. 7b).

304 Discussion

305 While previous single-electrode recordings have strongly advanced our understanding of the 306 neural correlates of perceptual decision-making, interesting dynamics in choice signals were lost 307 to necessity of averaging data across trials. With a few notable exceptions ${ }^{3,24}$, deploying the 308 statistical power of simultaneous multi-electrode recordings to track single-trial population 309 dynamics during choice behavior is a recent advance ${ }^{1,2,6}$. Even more recent work has leveraged the 310 power of brain-computer interfaces (BCIs) to study neural correlates of prediction, learning, and 311 multisensory integration (as reviewed in Golub et al. $2016^{25}$ ). In this study, for the first time, we 
312 probed moment-to-moment fluctuations in decision states using BCI-inspired closed loop

313 experiments that enabled neurally contingent stimulus control and made behavioral validation of

314 these fluctuations feasible (see Methods). We show that large fluctuations (up to several log units)

315 in a decoded decision variable in premotor and primary motor cortices are nearly instantaneously

$316(<100 \mathrm{~ms})$ predictive of choice. We captured neural correlates of changes of mind in the form of

317 robust changes in DV sign. The statistical regularities of these rare events match previous

318 psychophysical CoM findings. Finally, we showed that larger DV values are resistant to additional

319 pulses of sensory evidence, supporting the hypothesis that large DVs are associated with higher

320 commitment to an upcoming choice.

322 Importantly, the impressive choice prediction accuracy achieved in this study using a linear 323 decoder does not imply that the brain's decision formation process is also linear. In principle, such

324 a decoder could predict binary choices quite well even if the true neural process underlying

325 decision formation were nonlinear, depending on the form of the nonlinearity (see, e.g., Sussillo

326 et al. $2016^{26}$ for an example of a linear neural to kinematic decoder which only slightly

327 underperforms a more powerful nonlinear recurrent neural network). However, our linear DV is

328 tightly linked to choice behavior (e.g. Fig. 2c), showing that variations in DV magnitude

329 meaningfully track the ongoing process of decision formation despite the possible presence of 330 nonlinearities in the underlying neural mechanism.

332 Previous studies have described fluctuations in offline decoded decisions associated with changes

333 of mind ${ }^{1-3,6}$. Here we confirm and extend those observations with neurally contingent interrogation

334 of candidate CoM events, but we also find large, behaviorally relevant fluctuations even when the 
335 DV remains on one side of the discriminant hyperplane in non-CoM trials (e.g. Figs. 1e and 2b).

336 We wondered whether these DV fluctuations were related to stochastic variations in motion

337 strength of the stimulus on single trials. While across coherence levels the average motion energy

338 explains a large portion of DV variance (Extended Data Fig. 8a-b), our data shows that within

339 coherence stochastic fluctuations in the stimulus are not the dominant cause of DV fluctuations

340 (Extended Data Fig. 8c-d). Further experiments will be needed to address the source(s) of these

341 fluctuations and their relationship with fluctuations in other brain areas ${ }^{27}$ as well as other cognitive

342 processes including motor preparation and execution ${ }^{28,29}$, attention, motivation, and confidence.

344 In addition to validating the behavioral relevance of neurally detected DV fluctuations, our ability

345 to impose real-time task changes contingent upon them allowed us to show that neural and

346 behavioral responses to pulses of additional sensory evidence diminish when pulses are presented

347 at larger momentary DV values. These results, combined with the reduction in DV variability

348 observed over the course of single trials, suggest the presence of an absorbing decision bound in

349 these motor cortical neural populations, consistent with attractor dynamics in which the neural

350 population converges on a stable state as a decision is formed ${ }^{22,23}$.

352 The conceptual and technical innovation that enabled these findings is our ability to accurately 353 decode decision states in real time, which could bring the concept of cognitive prostheses ${ }^{30-33}$ much

354 closer to reality by providing another means of decoding subjects' goals for use as a flexible 355 prosthetic control signal. More broadly, the real-time closed loop approach demonstrated here may

356 be applicable not only to decision-making processes, but also to other cognitive phenomena such 357 as working memory and attention. 


\section{a}
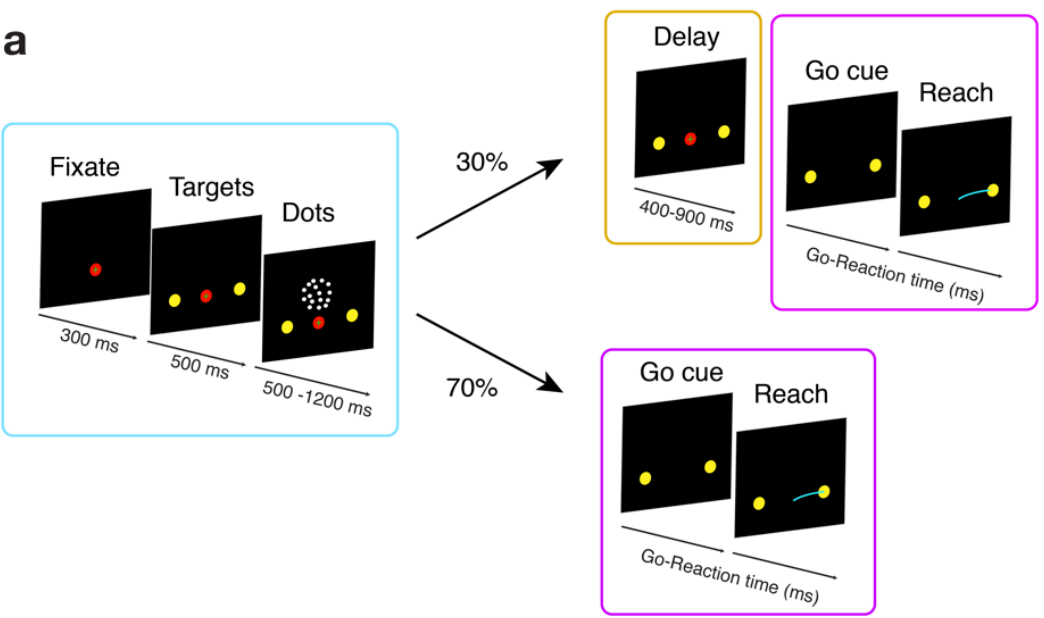

b

C

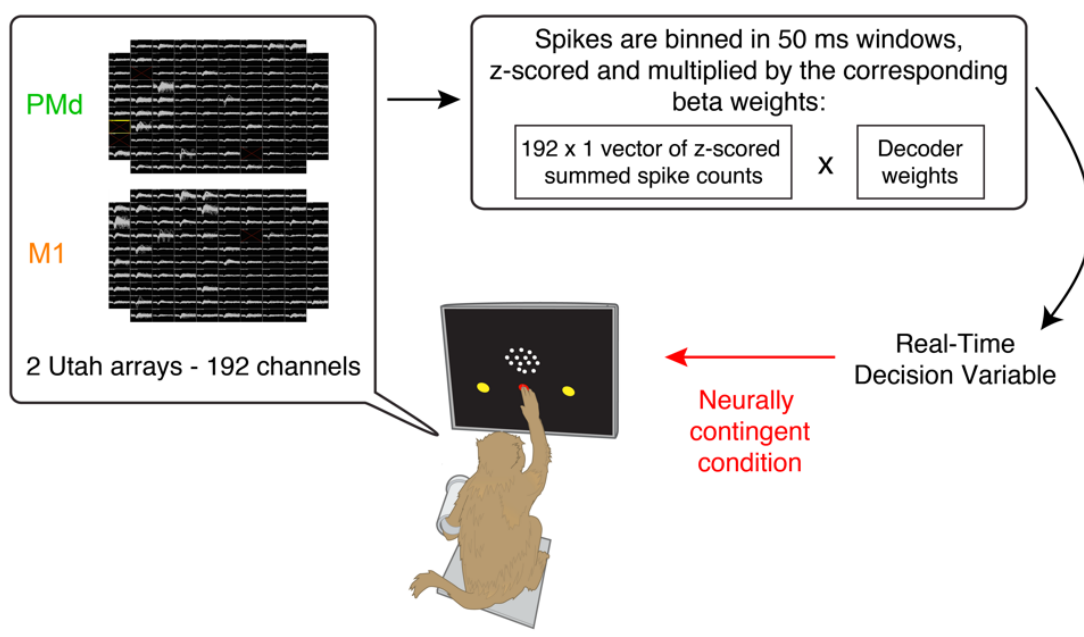

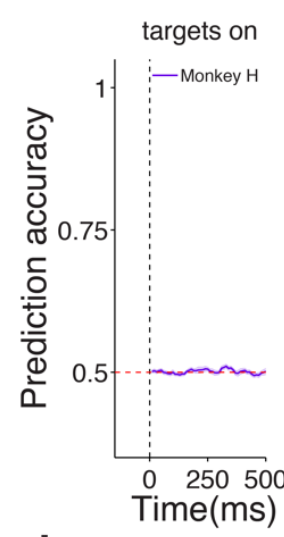

d
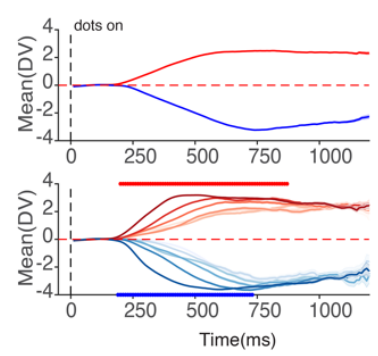

dots on

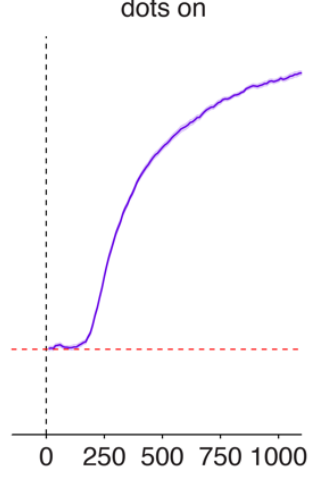

e

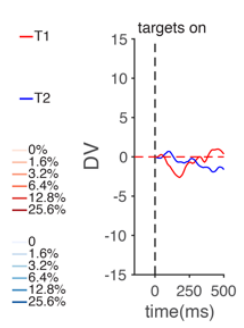

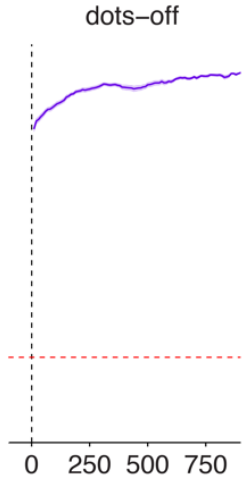

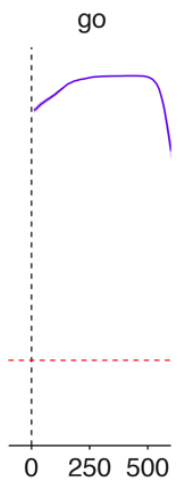

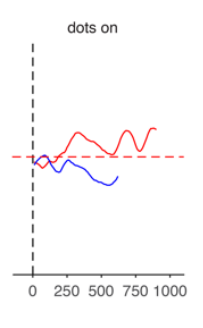
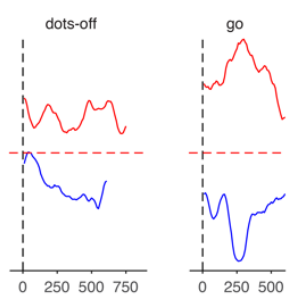
Figure 1. Setup and performance of real-time readout of decision states during a motion discrimination task.

a) Motion discrimination task. Trials began with the onset of a fixation point (FP) on the touchscreen. Once both eye and hand fixation were acquired, two targets appeared on the screen. The motion stimulus was shown after a short delay (500 ms) and lasted 500-1200 ms for the openloop trials. On $70 \%$ of the trials the dots offset was followed by the go cue (no delay period), while on the remaining $30 \%$ the subject was required to withhold a response for a random delay duration (400-900 ms). Decision states were continuously decoded during all epochs of the trial. Three different decoders were used during different trial epochs, shown by the different colored boxes (blue, yellow and purple; see Methods).

b) Real-time, closed-loop setup. Neural activity from 96-channel Utah Arrays was continuously

371 recorded and processed while monkeys performed the motion discrimination task. For monkey $\mathrm{H}$, 372 two Utah arrays implanted in PMd and M1 were used. For monkey F only one Utah array 373 implanted in PMd was utilized. During data collection, the recorded neural activity was binned, 374 summed, z-scored and projected onto a single dimension: a linear choice decoder. The result of 375 this operation was our real time read out of commitment, which could be used to stop the stimulus 376 presentation in a neurally contingent manner (red arrow), thereby closing the loop in the 377 experiment.

378 c) Choice prediction accuracy obtained from real-time, open-loop readout. Average 379 prediction accuracy (see Methods) over time \pm SEM for monkey H is plotted in purple. Prediction 380 accuracy is calculated for each time point aligned to four different events in the trial (targets onset, 381 dots onset, dots offset and go cue) using the real-time DV and quantified as the fraction of trials in 
382 which the classifier correctly predicted the monkey's upcoming choice. For logistic regression this

383 operation is equivalent to comparing the DV sign to the choice sign. Accuracy was calculated for

384 each session and averaged across sessions using a total of 16468 trials for monkey H.

385 d) Average Decision Variable traces during dots period. Top panel: Average DV during the

386 dots epoch for right (red) and left (blue) choices for monkey H. Bottom panel: Average DV sorted

387 by choice and stimulus coherence (correct trials only) for monkey H. Darker shades correspond to

388 higher stimulus coherence. Red and blue dots indicate timepoints for which coherence was

389 significant regressor of $\mathrm{DV}$ for $\mathrm{T} 1$ and $\mathrm{T} 2$ choices respectively (correct trials only, $\mathrm{p}<10^{-5}$

390 uncorrected). For monkey $\mathrm{H}$ coherence is a significant regressor of DV for at least one of the

391 choices for the period between [190, 870] ms aligned to dots onset.

392 e) Example DV traces captured during open loop trials. DV traces for two trials are plotted as

393 a function of time aligned to four different events: targets onset, dots onset, dots offset and go cue.

394 The trial in red led to a right choice whereas the trial in blue led to a left choice. Despite the stability

395 in DV sign for these two trials from $\sim 250 \mathrm{~ms}$ after dots onset until the end of the trial, strong

396 fluctuations in DV magnitude were observed in both cases, within and across epochs. 
a

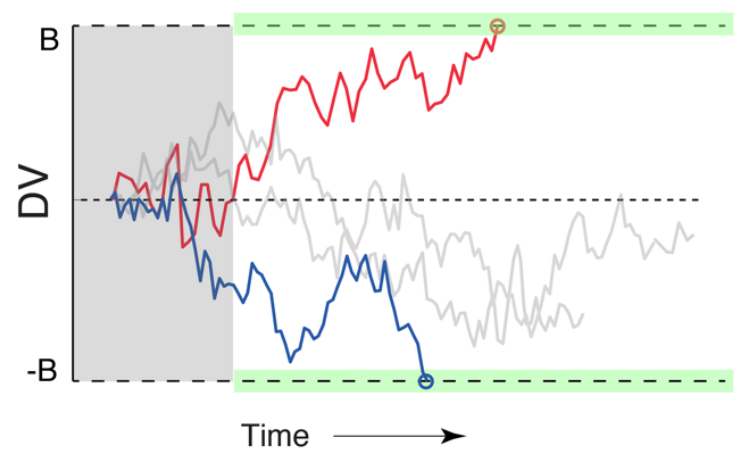

C

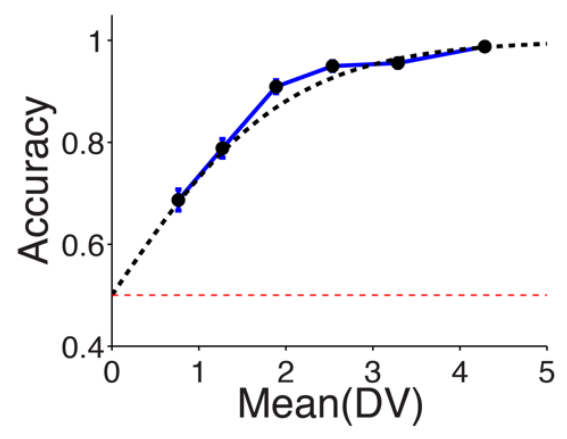

d

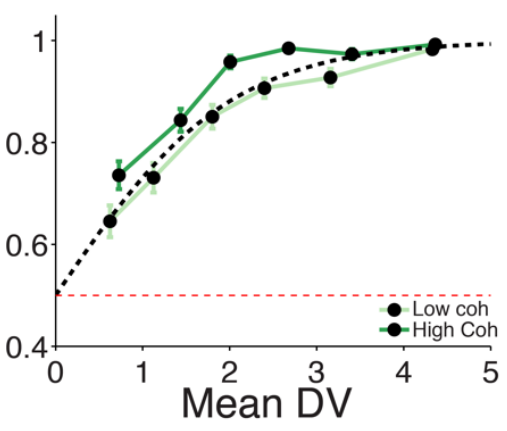

b

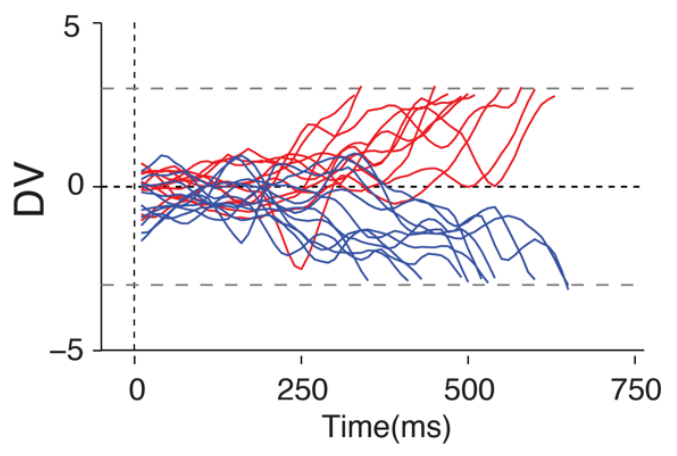

e

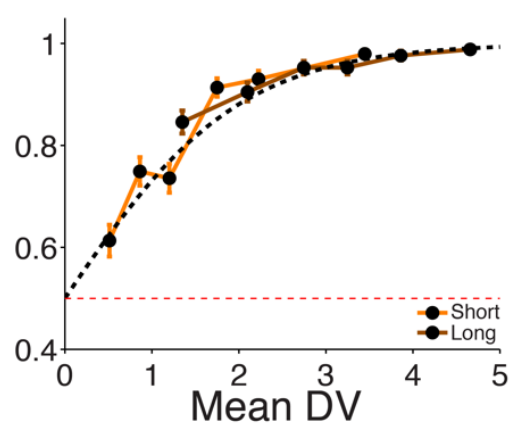

Figure 2. Choice likelihood can be accurately decoded in real-time across experimental conditions using only $50 \mathrm{~ms}$ of neural data. for DV magnitude (green shaded regions) were imposed and if reached, triggered the termination of the stimulus presentation. The subject was then immediately asked to report its decision. A 250 ms minimum stimulus duration was imposed (grey shaded region) to prevent random fluctuations in the beginning of the trial from triggering stimulus termination. If the boundary wasn't reached,

407 the stimulus was presented for a pre-selected random duration (500-1200 ms). Grey traces show 
409 trials that the decoder predicted would result in a right (left) choice. 5 different boundary values

410 were used on each experiment.

411 b) Example trials captured during the virtual boundary experiment. Real-time DV time

412 courses for example trials terminated using boundaries set at +3 and -3 DV units. Traces are

413 colored according to behavioral choice at the end of the trial: right choices in red and left choices

414 in blue. Data from one session from monkey H.

415 c) Prediction accuracy as a function of DV magnitude. Choice prediction accuracy for all trials

416 collected during virtual boundary experiment as a function of DV magnitude for monkey $\mathrm{H}$ is

417 shown in blue. Trials were split in 6 quantiles sorted by DV magnitude at termination. Prediction

418 accuracy and median DV magnitude were calculated and plotted separately for each quantile (blue

419 line with black symbols). Blue error bars show standard error of the mean for a binomial

420 distribution. Dashed black line shows predicted accuracy from log-odds equation and red dashed

421 line shows chance level. Data from 2973 trials from monkey H.

422 d) Prediction accuracy as a function of DV and stimulus coherence. Same data shown in c) but

423 having pre-sorted the trials by coherence (see Methods). Dark green trace shows high coherence

424 results and light green, low coherence results. Same conventions as in c).

425 e) Prediction accuracy as a function of DV and stimulus duration. Same data shown in c) but

426 having pre-sorted the trials by stimulus duration. Brown trace shows results for long trials and 427 orange trace results for short trials (see Methods). Same conventions as in c). 
a

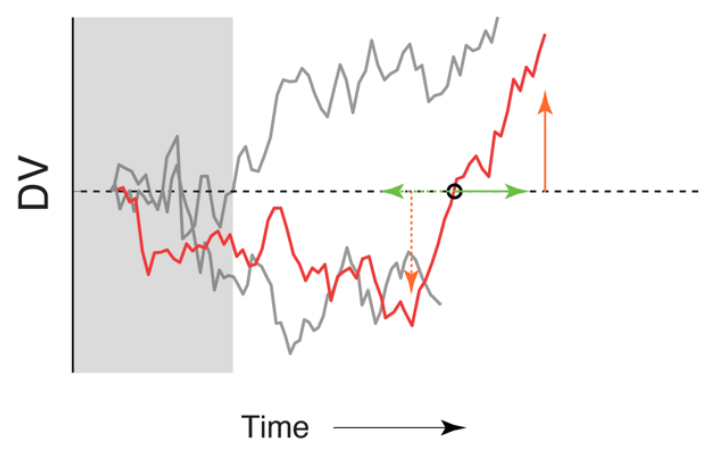

C

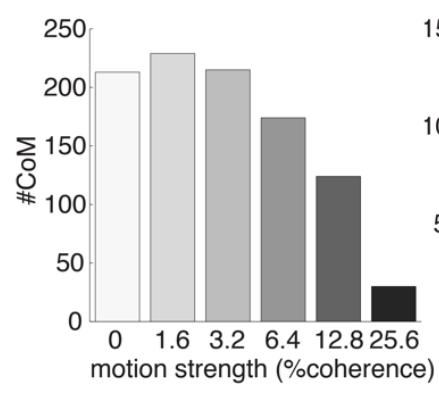

d

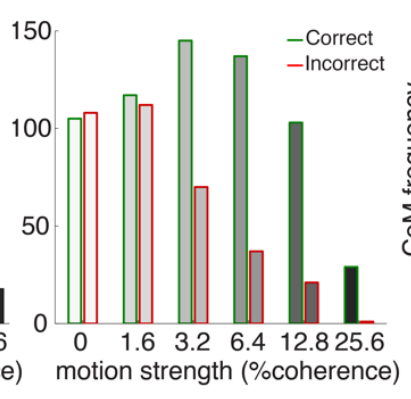

b

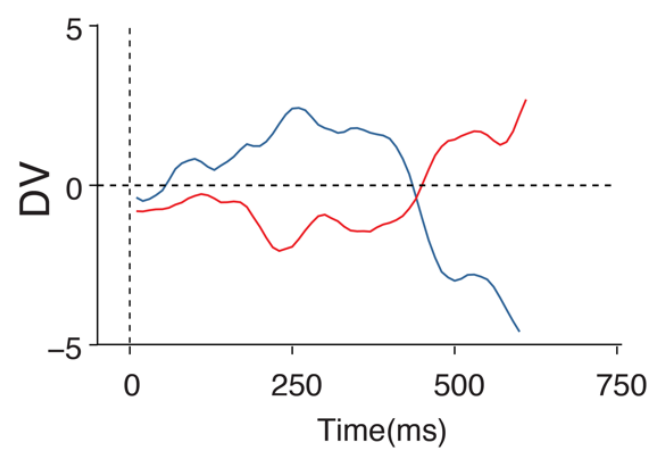

e

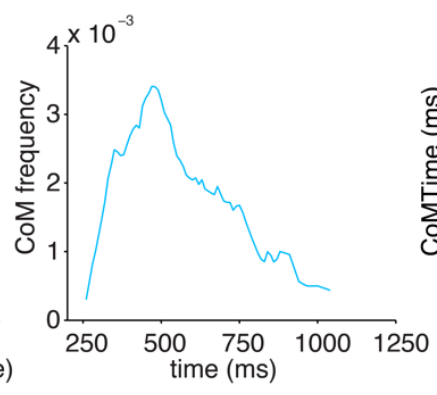

\section{f}

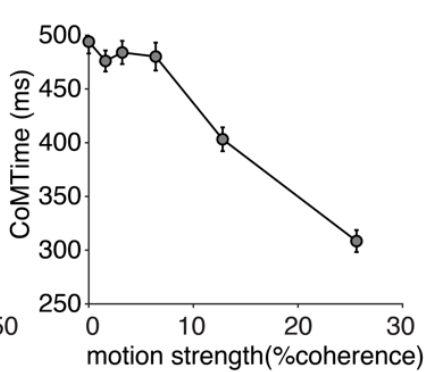

Figure 3 Putative changes of mind can be detected and validated in real time.

a) Schematic of the second closed loop experiment implemented in real time. The value and

434 history of the DV trace were tracked on each trial. If a 0 crossing (sign change in the DV) was

435 detected, the conditions required for termination were checked and termination was carried out if

436 the conditions were met (see Methods). In this example the conditions for temporal stability of DV

437 sign are depicted by the green horizontal arrows while the conditions for minimum DV deflection

438 before and after CoM are depicted by the orange arrows. Upon termination, the subject was

439 immediately asked to report its decision. A $250 \mathrm{~ms}$ minimum stimulus duration was imposed (grey

440 shaded region) such that random fluctuations in the beginning of the trial did not trigger stimulus 
441 termination. If the conditions were not met or if a 0 crossing was never detected, the stimulus

442 would be presented for a pre-selected random duration (500-1200 ms). Grey traces show cartoons

443 of trials for which the 0 crossings would not meet the criteria while the red the trace shows a

444 terminated trial that was predicted to lead to a rightward choice. One set of criteria for CoM validity

445 was used in each session (Extended Data. Table 4).

446 b) Example trials captured during the CoM experiment. Real-time DV time courses for 2

447 example trials with a putative CoM terminated after conditions were met (minimum DV pre and

448 post CoM: 2 and minimum period of sign stability pre and post CoM: $150 \mathrm{~ms}$ ). Traces are colored

449 according to behavioral choice at the end of the trial: right choices in red and left choices in blue.

450 Two trials from one session from monkey $\mathrm{H}$.

451 c) CoM frequency as a function of coherence. Total number of CoMs detected for each 452 coherence for monkey $\mathrm{H}$.

453 d) CoM frequency as a function of coherence and direction. Total number of CoMs detected

454 for each coherence and direction for monkey H. Red bars correspond to erroneous CoMs and green

455 bars to corrective CoMs.

456 e) CoM frequency as a function of time in the trial. Frequency of CoMs detected as a function

457 of time during stimulus presentation for monkey H. Because only CoMs that would have resolved

458 by 250 msec after stimulus onset were considered, there is an edge effect with CoM frequency

459 briefly increasing between $\sim 250-450 \mathrm{msec}$ after which it declines.

460 f) CoM time as a function of coherence. Average CoM time (defined as the zero crossing for

461 each CoM trial) is plotted as a function of stimulus coherence. Error bars show s.e.m across trials

462 for each condition. CoM time was negatively correlated with stimulus coherence $\left(\mathrm{p}=1.8 \times 10^{-17}\right)$ 
a

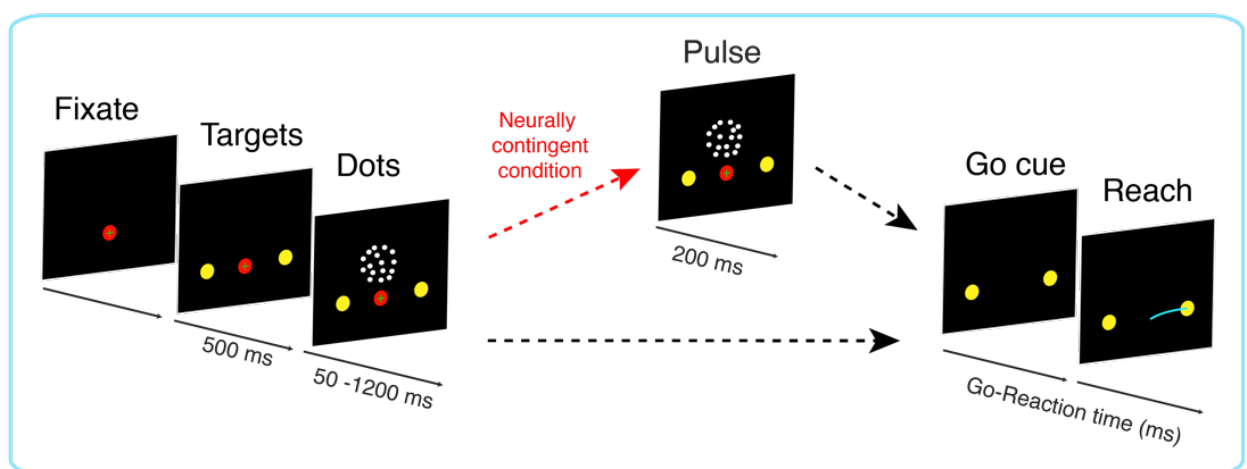

b

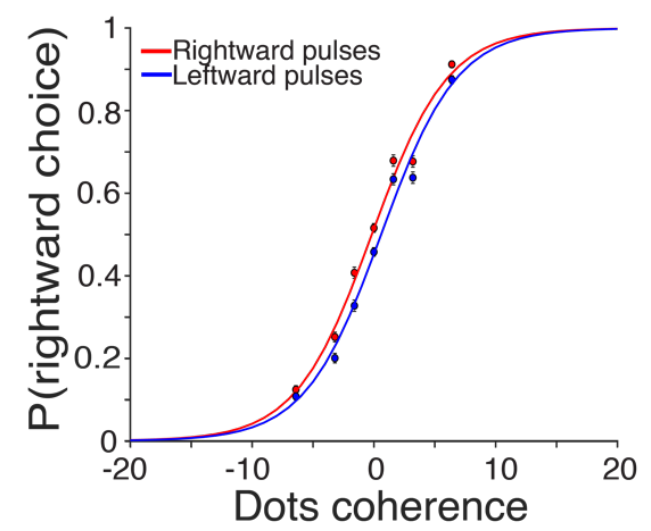

d

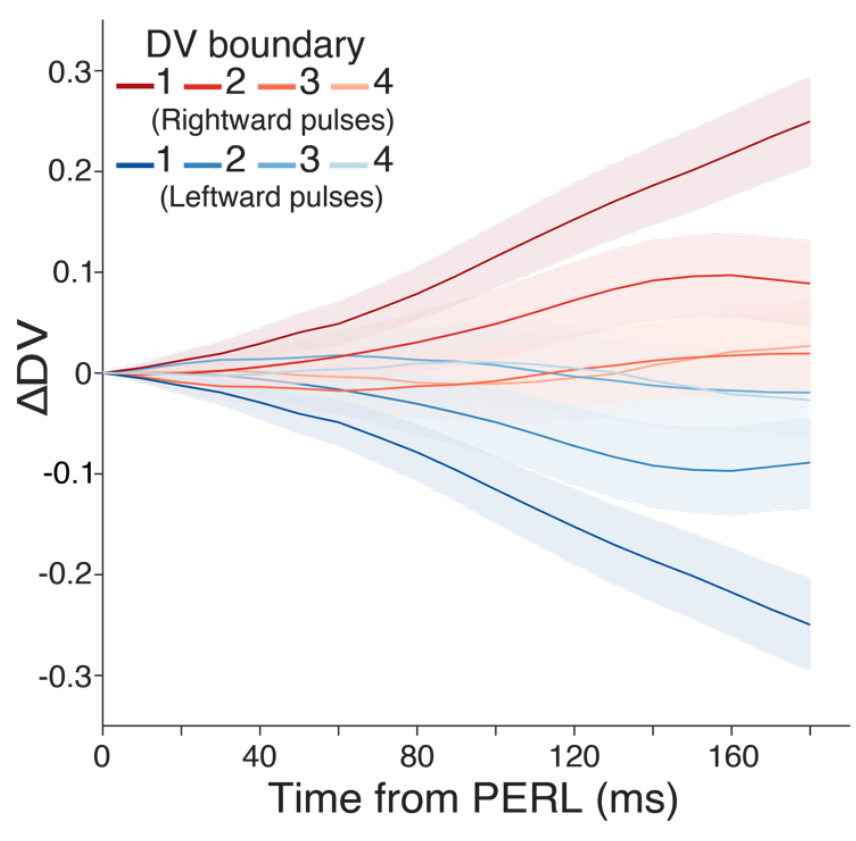

C

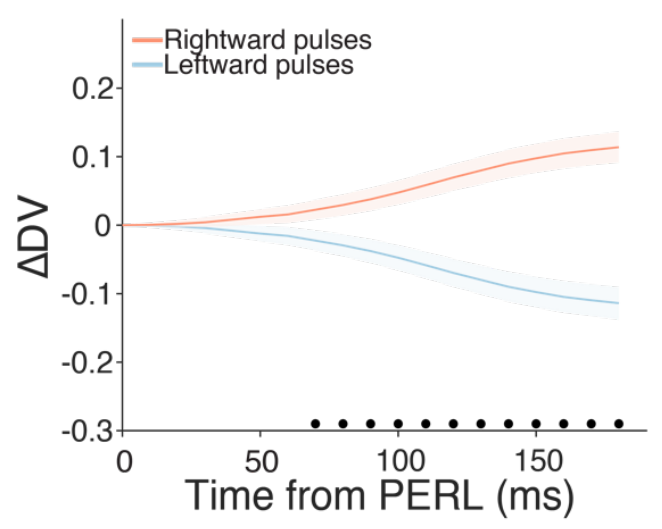

e
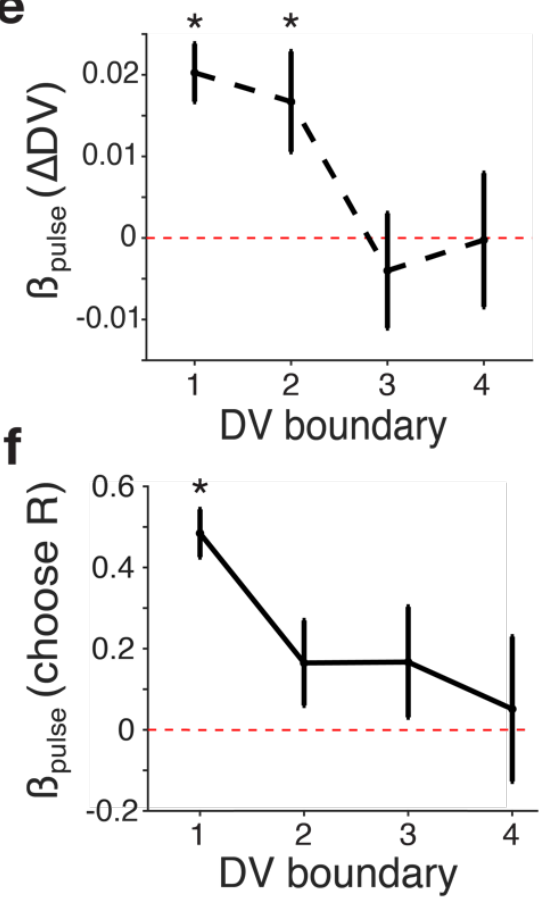
Figure 4. Neurally triggered pulses of motion evidence nonlinearly bias both choice behavior and DV.

a) Motion pulse task. As in the motion discrimination task, trials began with the onset of a fixation point (FP) on the touchscreen. Once both eye and hand fixation were acquired, two targets appeared. The motion stimulus was shown after a short delay (500 ms) and a maximum stimulus

472 duration was randomly assigned from 500-1200 ms. Virtual boundaries for DV magnitude were

473 imposed (randomly assigned to integer values from 1-4) and if reached, triggered a 200-ms pulse

474 of additive dots coherence, randomly assigned to be rightward or leftward on each trial $( \pm 2 \%$

475 coherence for monkey $\mathrm{H}$ ), followed immediately by termination of the dots stimulus. A $50 \mathrm{~ms}$

476 minimum stimulus duration was imposed to ensure a minimum total stimulus duration of $250 \mathrm{~ms}$.

477 If the DV boundary wasn't reached, the dots stimulus was presented for a pre-selected random 478 duration (500-1200 ms). Dots offset was followed by the go cue. Decision states were continuously

479 decoded using the dots period decoder during all epochs of the trial (blue box, see Methods).

480 b) Psychometric functions for pulse trials. Curves were fit using logistic regression on choice 481 with signed stimulus coherence and pulse direction as predictors, plus an intercept term. Data 482 points show mean proportion of rightward choices for each stimulus coherence, \pm s.e.m. The pulse 483 effect is equivalent to changing the overall stimulus coherence by $0.384 \%$ (standard error $4840.0514 \%, \mathrm{p}=8.38 \mathrm{E}-14)$. Data from 9614 rightward and 9523 leftward pulse trials from monkey $485 \mathrm{H}$.

486 c) Average change in post-pulse DV from estimated Pulse Evidence Representation Latency

487 (PERL), mean subtracted. $\triangle \mathrm{DV}$ is the difference in the DV at each time point from the DV at 
the PERL (170 ms for monkey H; see Methods). The mean $\triangle \mathrm{DV}$ across pulse directions in each time bin has been subtracted for visualization. Shaded error bars correspond to mean \pm s.e.m. Black dots indicate time bins in which $\Delta \mathrm{DV}$ is significantly different for trials with pulses in opposite

491 directions (false discovery rate 0.05 ). Data from same trials as $\mathbf{b}$ ). correspond to smaller DV boundary magnitudes. Data from monkey H, minimum 1507 trials per condition shown.

e) Pulse coefficients from linear regression on $\triangle D V$ slope for each DV boundary. $\Delta D V$ slope is the single-trial slope of the $\triangle \mathrm{DV}$ from PERL to either the animal's median go-reaction time or

$498150 \mathrm{~ms}$ prior to movement onset, whichever came first (as shown in b)). Multiple linear regression was performed separately on $\triangle \mathrm{DV}$ slope for trials at each DV boundary with the following

500 predictors: signed dots coherence, pulse onset time, pulse direction, pulse onset time * pulse

501 direction, plus an intercept term. Data points and error bars represent the coefficient for pulse 502 direction for trials at each DV boundary, \pm s.e.m.; asterisks denote significantly nonzero 503 coefficients at $95 \%$ confidence. Data from same trials as $\mathbf{d}$ ).

\section{4 f) Pulse coefficients from logistic regression on choice for each DV boundary. Logistic}

505 regression was performed separately on the probability of a rightward choice for trials at each DV 506 boundary with the following predictors: signed dots coherence, pulse onset time, pulse direction, 507 pulse onset time * pulse direction, plus an intercept term. Data points and error bars represent the 508 coefficient for pulse direction for trials at each DV boundary, \pm s.e.m.; asterisks denote 509 significantly nonzero coefficients at $95 \%$ confidence. Data from same trials as d). 


\section{$\Delta \mathrm{DV}=$ Within trial variability}

a

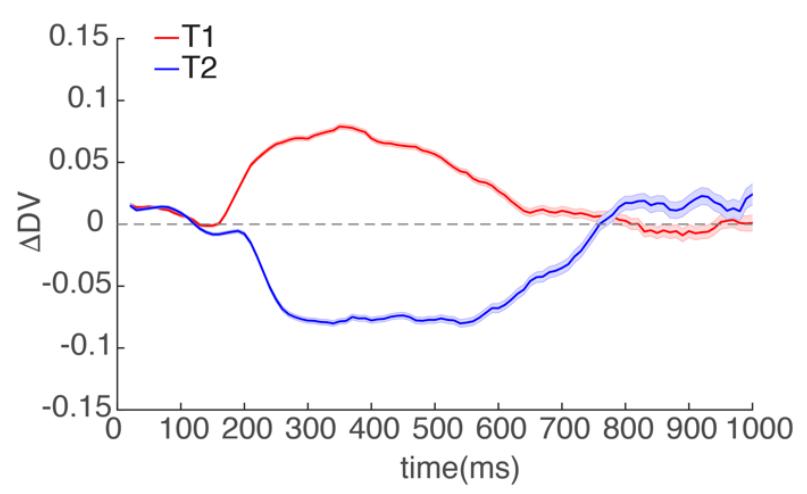

b

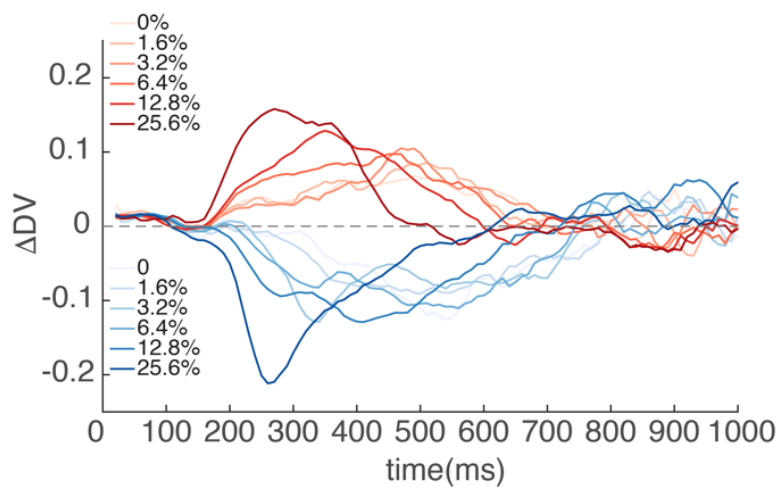

Figure 5. Within trial DV variability decreases over time for long duration stimuli.

516 for each trial as the difference between consecutive DV estimates spaced out by $10 \mathrm{~ms}$. Traces

517 show average $\Delta \mathrm{DV}+/$ - s.e.m for right choices (red trace) and left choices (blue trace) during

518 stimulus presentation. $\triangle \mathrm{DV}$ initially starts increasing around the expected stimulus latency (170

$519 \mathrm{~ms}$ ) but progressively decreases for long (>600 ms) stimulus presentations.

521 as in a) but with DV derivative averaged separately for each choice and motion coherence level

522 (correct trials only). Right choices are plotted in red and left choices in blue as in a). Darker traces

523 correspond to stronger coherences. 


\section{Extended Data}

\begin{tabular}{|c|c|c|c|c|c|c|}
\hline \multicolumn{7}{|c|}{ Logistic Regression on Choice } \\
\hline \multirow[b]{2}{*}{ Predictor } & \multicolumn{3}{|c|}{ Monkey H } & \multicolumn{3}{|c|}{ Monkey F } \\
\hline & $\begin{array}{l}\text { Beta } \\
\text { Value } \\
\end{array}$ & $95 \%$ CI & p-value & $\begin{array}{l}\text { Beta } \\
\text { Value } \\
\end{array}$ & $95 \% \mathrm{CI}$ & p-value \\
\hline Bias & -0.1613 & $\begin{array}{c}{[-0.2987,-} \\
0.02381]\end{array}$ & 0.02147 & -0.1197 & $\begin{array}{l}{[-0.2413,} \\
0.001806]\end{array}$ & 0.0535 \\
\hline Coherence & 2.747 & $\begin{array}{l}\text { [2.268 } \\
, 3.227] \\
\end{array}$ & $2.757 \mathrm{e}-29$ & 1.885 & 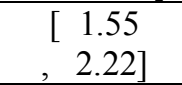 & $2.912 \mathrm{e}-28$ \\
\hline DV Termination & 2.073 & $\begin{array}{r}{[1.779} \\
, 2.367]\end{array}$ & $1.887 \mathrm{e}-43$ & 1.708 & 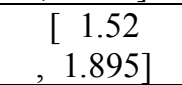 & $1.626 \mathrm{e}-71$ \\
\hline DV Diff & 0.2989 & $\begin{array}{c}{[0.0488} \\
0.5491]\end{array}$ & 0.01916 & 0.577 & $\begin{array}{c}{[0.4062} \\
0.7478]\end{array}$ & $3.586 \mathrm{e}-11$ \\
\hline Stimulus duration & 0.1199 & $\begin{array}{c}-0.01489, \\
0.2548]\end{array}$ & 0.08125 & 0.07474 & $\begin{array}{c}{[-0.04713,} \\
0.1966]\end{array}$ & 0.2293 \\
\hline $\begin{array}{l}\text { Stimulus } \\
\text { duration* } \\
\text { Stimulus direction }\end{array}$ & -0.02803 & $\begin{array}{c}{[-0.2053} \\
0.1492]\end{array}$ & 0.7566 & 0.0466 & $\begin{array}{c}{[-0.1132} \\
0.2064]\end{array}$ & 0.5677 \\
\hline
\end{tabular}

Extended Data Table 1 - Coefficients obtained from logistic regression on choice - virtual

\begin{tabular}{|c|c|c|c|c|c|c|}
\hline \multicolumn{7}{|c|}{ Logistic Regression on Choice after CoM } \\
\hline \multirow[b]{2}{*}{ Predictor } & \multicolumn{3}{|c|}{ Monkey $\mathrm{H}$} & \multicolumn{3}{|c|}{ Monkey F } \\
\hline & $\begin{array}{l}\text { Beta } \\
\text { Value }\end{array}$ & $95 \%$ CI & p-value & $\begin{array}{l}\text { Beta } \\
\text { Value }\end{array}$ & $95 \%$ CI & p-value \\
\hline Bias & -0.2876 & $\begin{array}{c}{[-0.4658,-} \\
0.1093]\end{array}$ & 0.001568 & -0.192 & $\begin{array}{c}-0.3773,- \\
0.00662]\end{array}$ & 0.04235 \\
\hline Coherence & 1.304 & 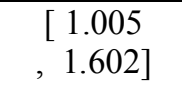 & $1.189 \mathrm{e}-17$ & 1.284 & $\begin{array}{c}{\left[\begin{array}{c}0.96 \\
, \\
1.608\end{array}\right]} \\
\end{array}$ & $8.028 \mathrm{e}-15$ \\
\hline DV Termination & 1.67 & 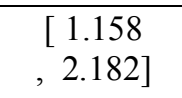 & $1.623 \mathrm{e}-10$ & 2.46 & $\begin{array}{l}{\left[\begin{array}{l}1.872 \\
, 3.049\end{array}\right]}\end{array}$ & $2.51 \mathrm{e}-16$ \\
\hline DV max opposite & 0.06967 & $\begin{array}{c}-0.4461, \\
0.5854]\end{array}$ & 0.7912 & -0.2387 & $\begin{array}{c}-0.8959 \\
0.4185]\end{array}$ & 0.4766 \\
\hline $\begin{array}{l}\text { Time after CoM * } \\
\text { sign(DV } \\
\text { Termination) }\end{array}$ & 0.7065 & $\begin{array}{c}{[0.2317} \\
, 1.181]\end{array}$ & 0.003544 & 0.02265 & $\begin{array}{c}{[-0.3835} \\
0.4288]\end{array}$ & 0.913 \\
\hline $\begin{array}{l}\text { Time before CoM } \\
\text { * sign(DV max } \\
\text { opposite) }\end{array}$ & 0.7471 & $\begin{array}{c}{[0.3083} \\
, 1.186]\end{array}$ & 0.0008467 & -0.1427 & $\begin{array}{c}{[-0.6394} \\
0.3539]\end{array}$ & 0.5732 \\
\hline
\end{tabular}



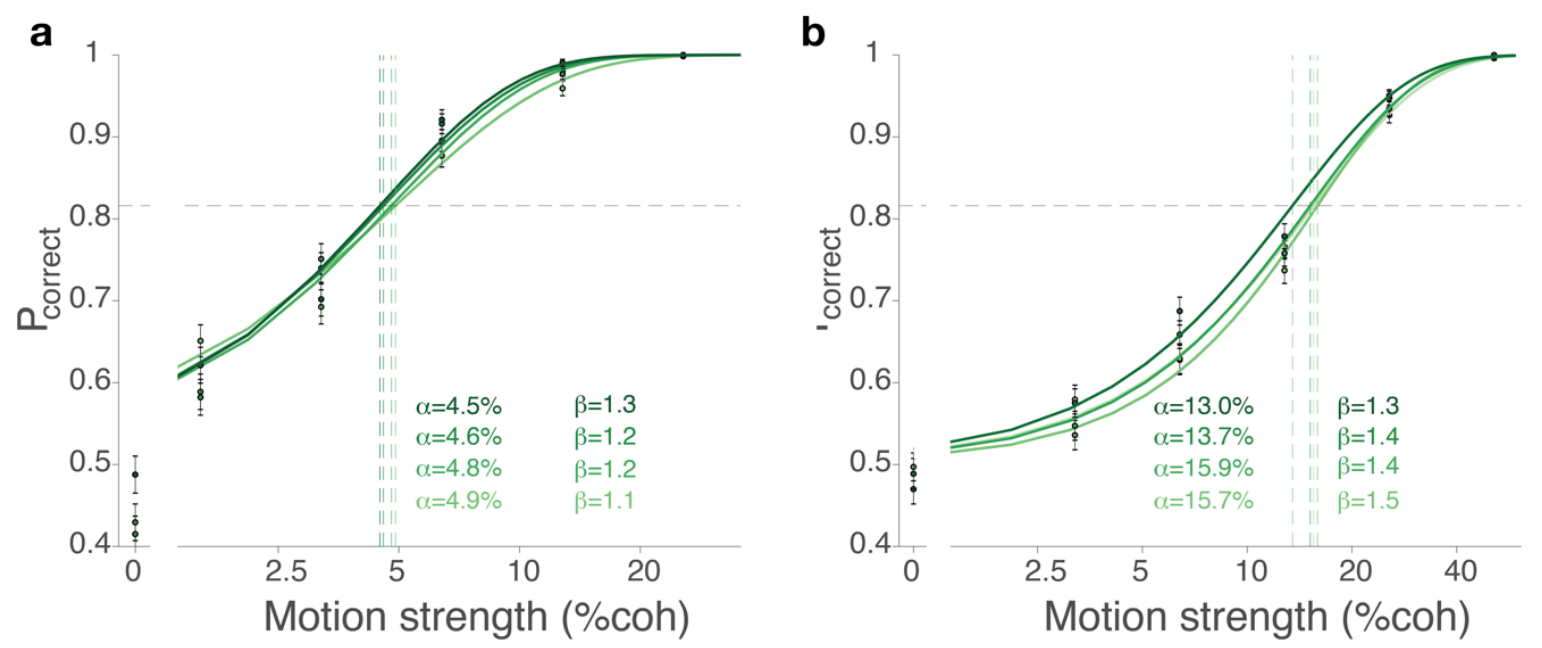

Extended Data Figure 1 - Behavioral performance - Variable duration task. sorted for stimulus duration in 4 quartiles from long (dark green curve) to short (light green curve).

547 Data from each quartile were fit separately by a Weibull curve. Inset shows fit parameters for each 548 quartile. Data from 12516 open loop trials. Stimulus duration quartiles: Q1: [0.500, 0.574] s Q2: $549 \quad[0.574,0.680] \mathrm{s}$ Q3[0.680, 0.827] s Q4: [0.827, 1.200] s. 
a

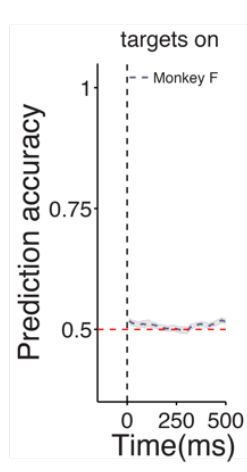

C

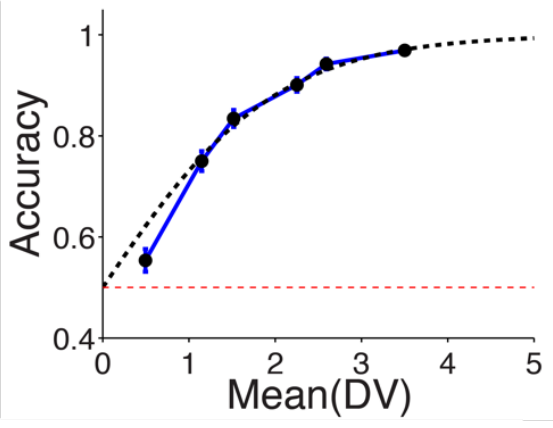

$\mathbf{f}$

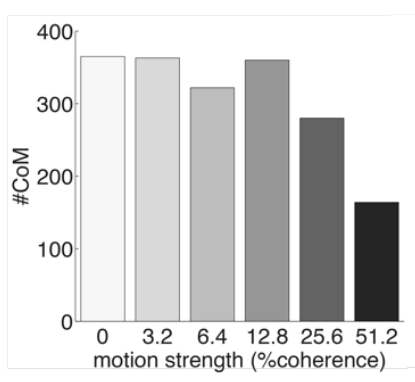

j

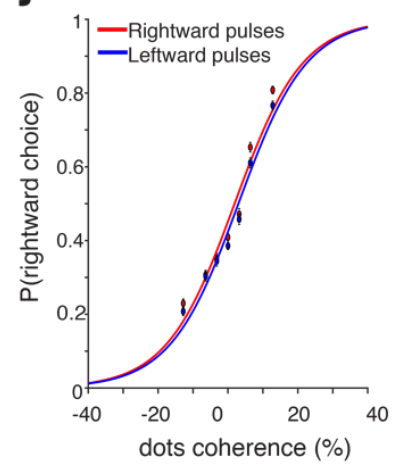

g

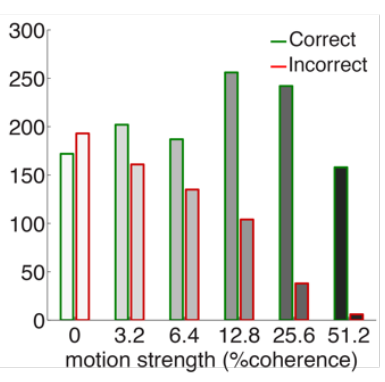

k

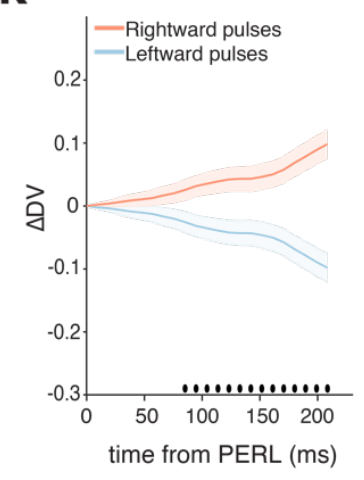

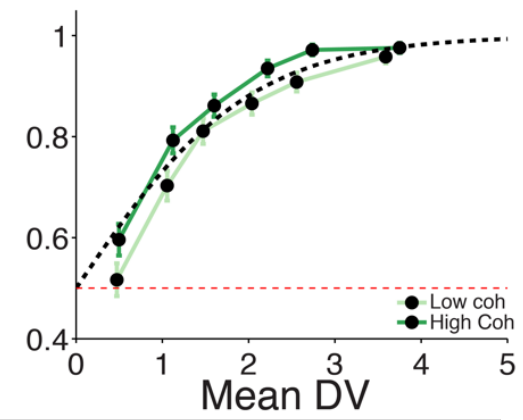

h

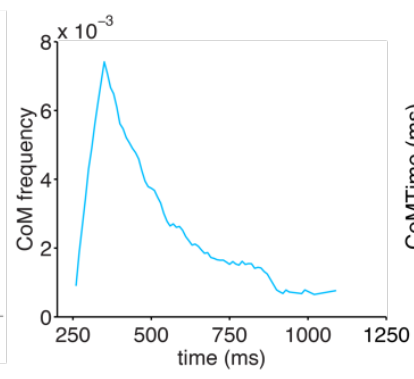

I

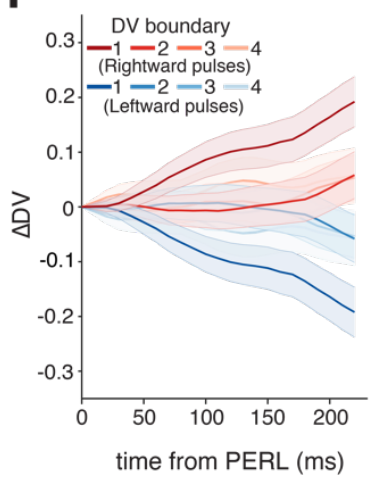

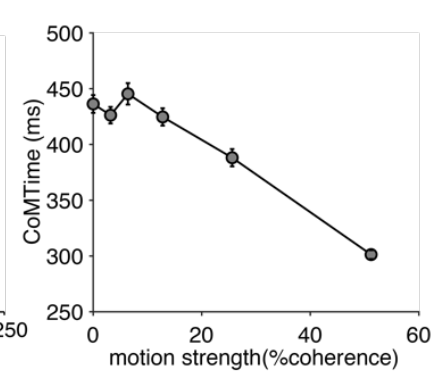

b

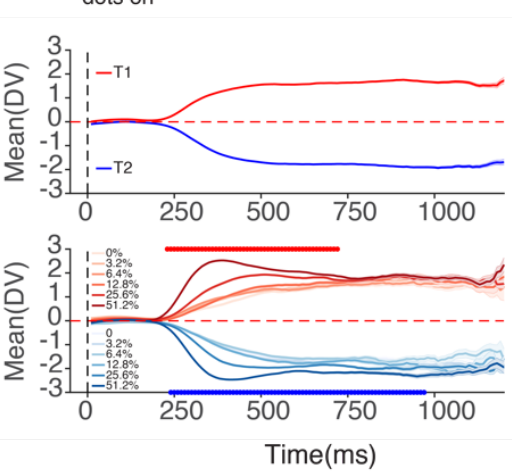

e

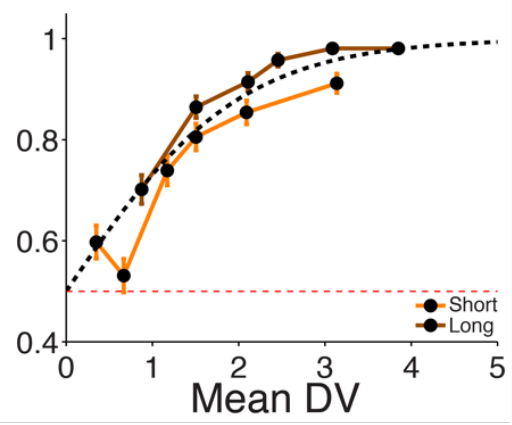

i

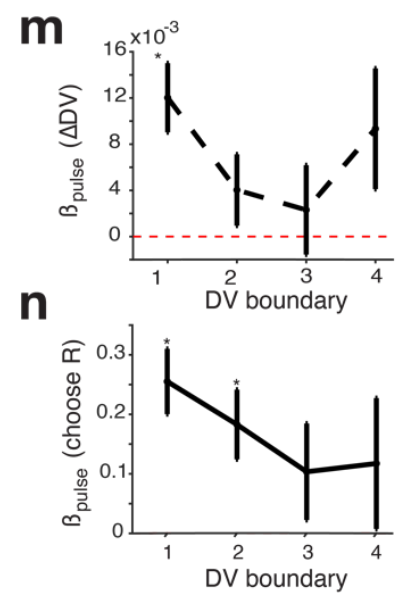


b) Average Decision Variable traces during dots period. Same as Figure 1d for monkey F. For

563 monkey $\mathrm{F}$ coherence is a significant regressor of DV for at least one of the choices for the period

564 between $[230,970] \mathrm{ms}$ aligned to dots onset.

565 c) Prediction accuracy as a function of DV magnitude. Same as Figure 2c for monkey F. Data 566 from 2518 trials from monkey F.

567 d) Prediction accuracy as a function of DV and stimulus coherence. Same data shown in c but 568 having pre-sorted the trials by coherence (see Methods). Dark green trace shows high coherence 569 results and light green, low coherence results. Same conventions as in c.

570 e) Prediction accuracy as a function of DV and stimulus duration. Same data shown in c) but

571 having pre-sorted the trials by stimulus duration. Brown trace shows results for long trials and 572 orange trace results for short trials (see Methods). Same conventions as in c).

573 f) CoM frequency as a function of coherence. Same as Figure 3c for monkey F.

574 g) CoM frequency as a function of coherence and direction. Same as Figure 3d for monkey F.

575 h) CoM frequency as a function of time in the trial. Same as Figure 3e for monkey F.

576 i) CoM time as a function of coherence. Same as Figure $3 f$ for monkey F. CoM time was 577 negatively correlated with stimulus coherence $\left(\mathrm{p}=3.0 \times 10^{-30}\right)$ 
578 j) Psychometric functions for pulse trials. Same as Figure $\mathbf{4 b}$ for monkey F. The pulse effect is

579 equivalent to changing the overall stimulus coherence by $0.545 \%$ (standard error $0.146 \%, \mathrm{p}=$

580 1.95E-4). Data from 10370 rightward and 9967 leftward pulse trials.

581 k) Average change in post-pulse DV from estimated Pulse Evidence Representation Latency

582 (PERL), mean subtracted. Same as Figure 4c for monkey F. PERL $=180 \mathrm{~ms}$. Data from same

583 trials as $\mathbf{j})$.

584 I) Average change in post-pulse DV for each DV boundary, mean subtracted. Same as Figure

585 4d for monkey F. Minimum 1731 trials per condition shown.

586 m) Pulse coefficients from linear regression on $\Delta$ DV slope for each DV boundary. Same as

$587 \quad$ Figure 4e for monkey F.

588 n) Pulse coefficients from logistic regression on choice for each DV boundary. Same as Figure

589 4f for monkey $F$.

590 

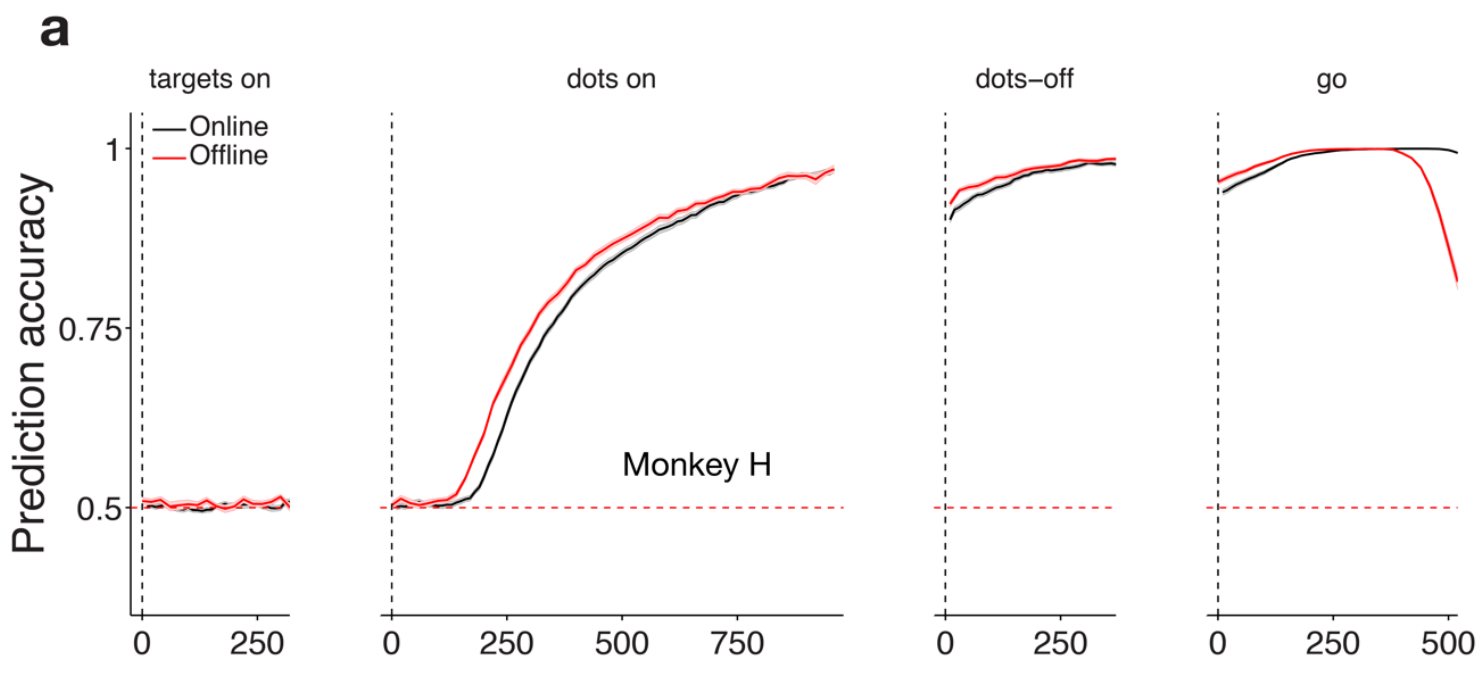

b
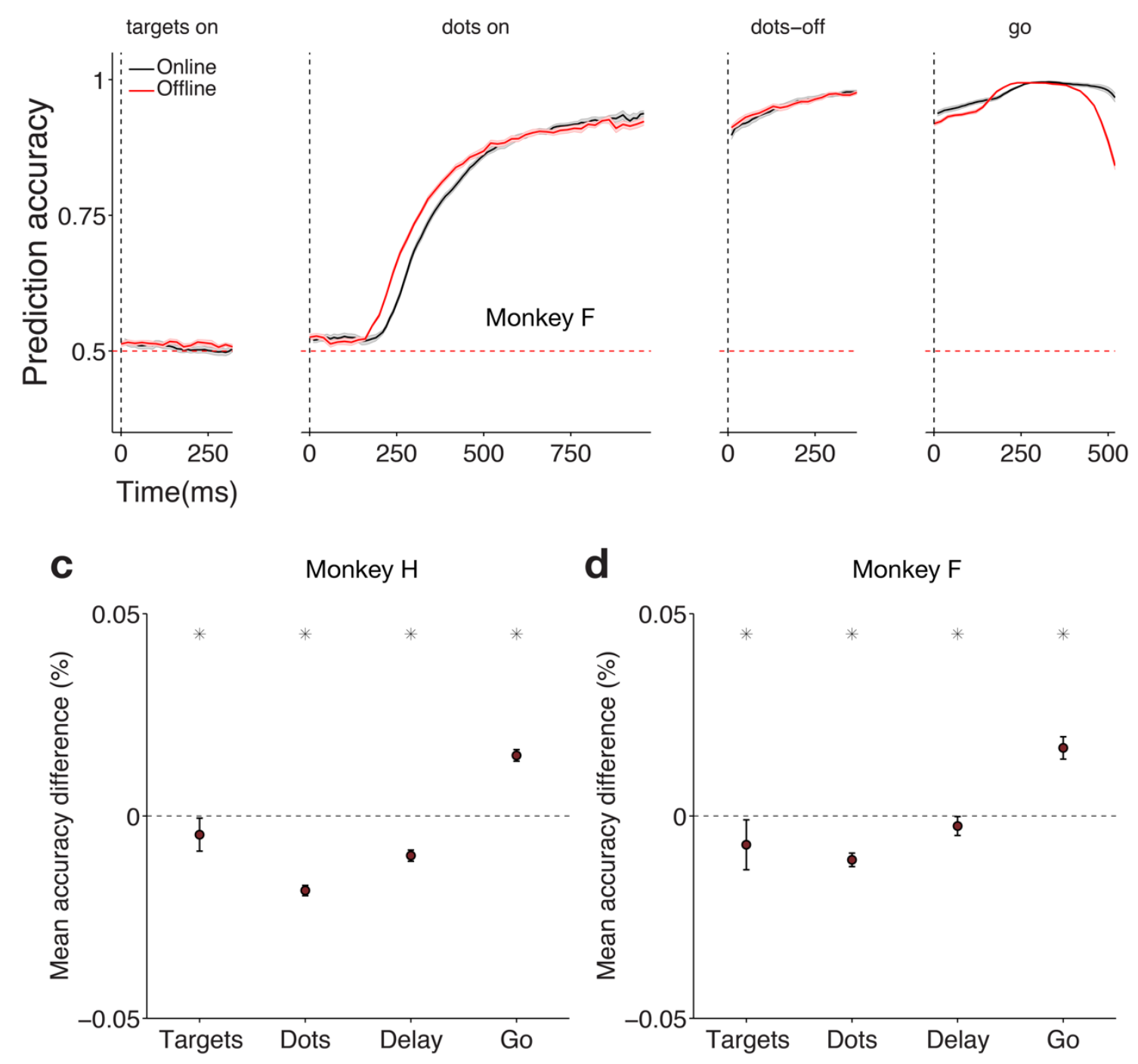
Extended Data Figure 3 - Prediction accuracy online, offline and as a function of stimulus

593 coherence.

594

595 a) Online and Offline classifiers result in similar performance for targets, dots delay and post-go epochs for monkey H. Average prediction accuracy (see Methods) over time \pm SEM

597 (across sessions) for monkey H. Online / offline classifier results are plotted in black / red. Data 598 in black is re-plotted from Figure 2a. Prediction accuracy is very similar online and offline across 599 the trial (see c)).

600 b) Online and Offline classifiers result in similar performance for targets, dots delay and 601 post-go epochs for monkey F. Same as a but for monkey F. Same conventions apply.

602 c) Summary of performance difference between online and offline classifiers within each 603 epoch for monkey H. Average performance difference between online and offline classifiers 604 (accuracy difference in percentage correct) for each of the epochs plotted in a). Positive number numbers correspond to better online classifier performance and negative numbers to better offline 606 classifier performance. Black asterisks correspond to windows for which the differences were 607 significantly larger than zero (Wilcoxon signed-rank test, $\mathrm{P}<0.001$ ).

609 epoch for monkey F. Same as c) for monkey F. For both monkeys c) and d) the difference of 610 choice prediction accuracies between the online and the offline classifiers was small and negative 611 for the target, dots and delay epochs (between $-0.2 \%$ and $-1.9 \%$ ). In contrast, for the post-go 612 period, the difference in prediction accuracies was slightly positive $(1.5 \%$ and $1.7 \%$ for monkey $613 \quad \mathrm{H}$ and F respectively). 


\section{Monkey $\mathrm{H}$}
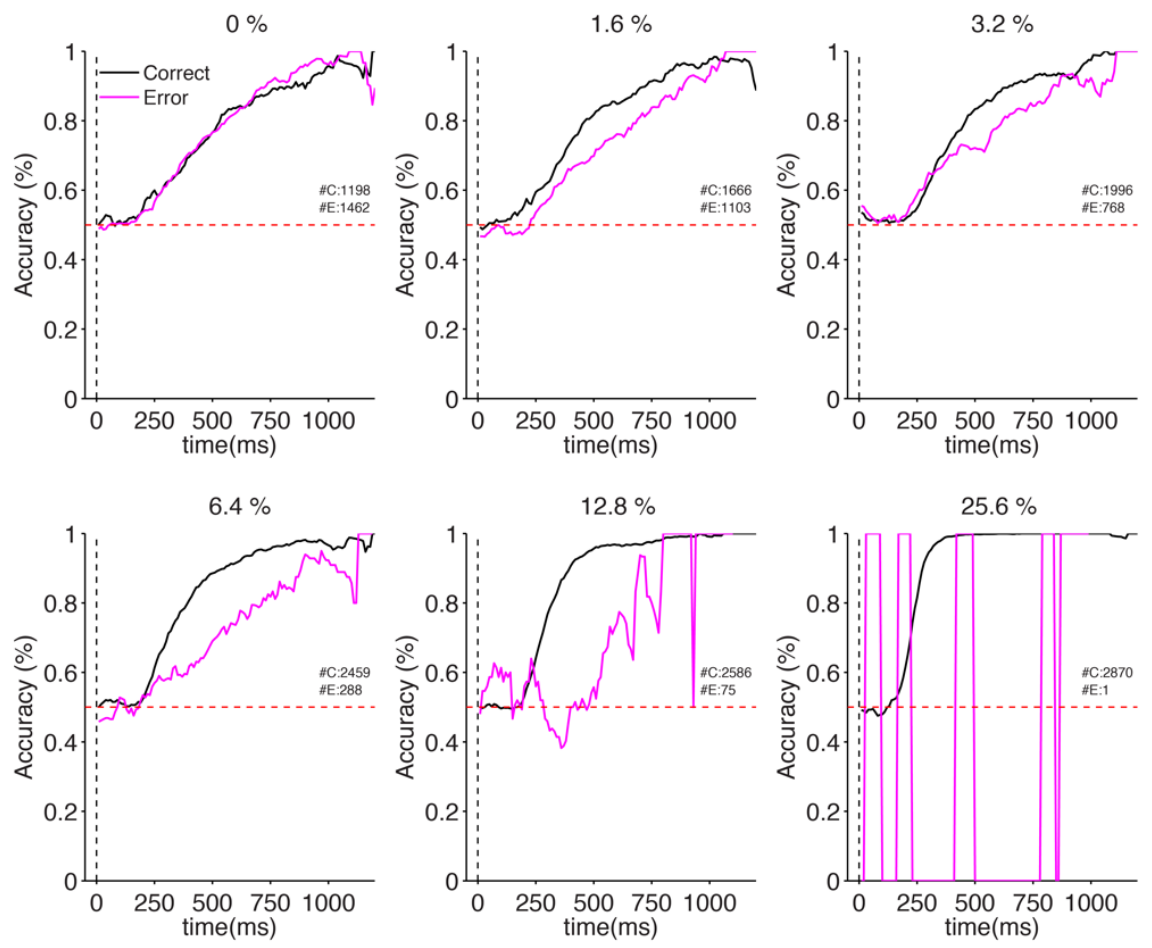

\section{Monkey F}
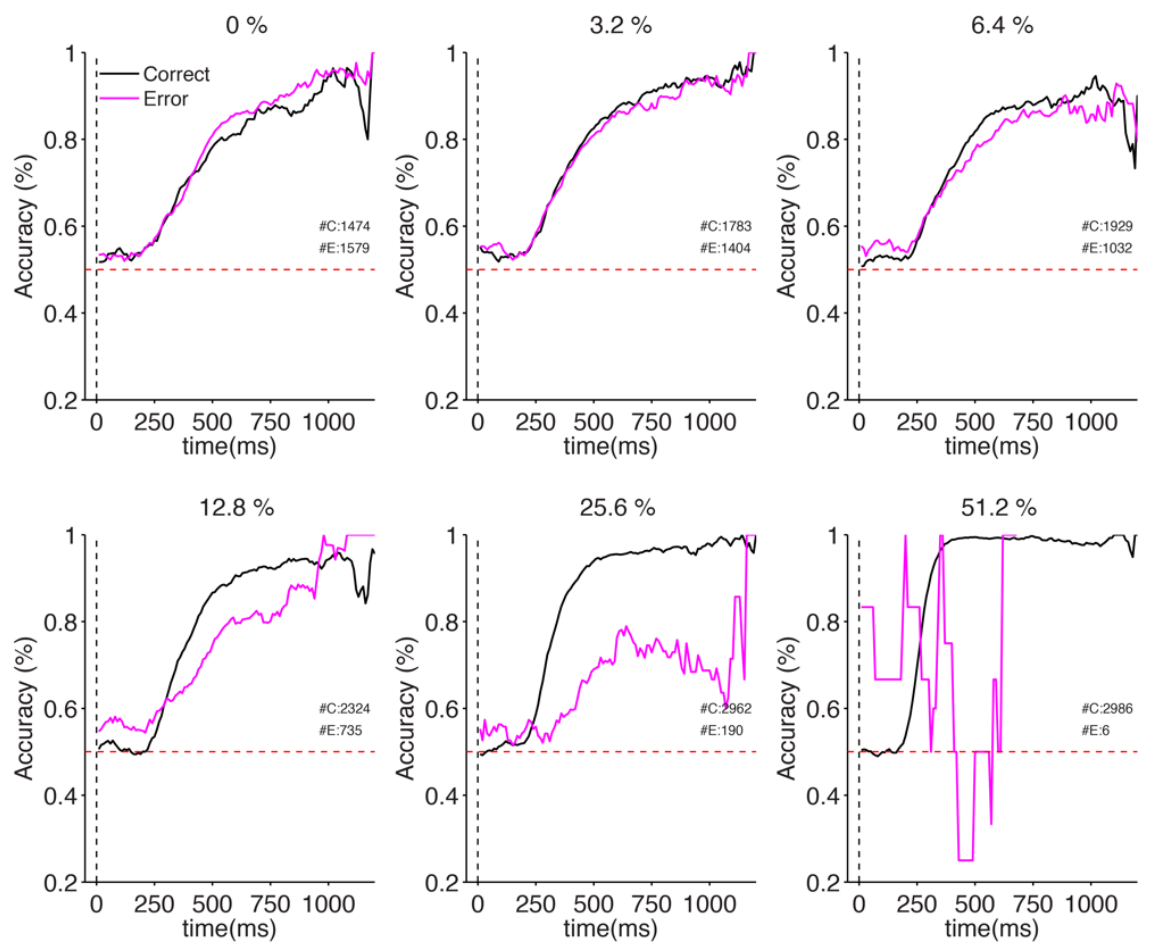
616 Extended Data Figure 4 - Choice prediction accuracy for correct and incorrect trials as a

617 function of coherence.

619 Choice prediction accuracy obtained from real-time readout for correct and incorrect trials for each

620 level of coherence. Prediction accuracy during dots epoch for each coherence level is plotted for

621 correct (black) and error (magenta) trials. Red dashed line corresponds to chance level. Insets show

622 total number of Correct $(\mathrm{C})$ and Error $(\mathrm{E})$ trials used in the analysis. Data for monkey $\mathrm{H}$ and $\mathrm{F}$ are

623 shown in top and bottom panels, respectively. Mean prediction accuracy for error trials after neural

624 latency (180 ms after stimulus presentation) is outside (and lower than) the 95\% CI for correct

625 trials for $1.6 \%, 3.2 \%, 6.4 \%, 12.8 \%$ and $25.6 \%$ coherences for monkey $\mathrm{H}$ and for $12.8 \%, 25.6 \%$

626 and 51.2\% coherences for monkey F - 1000 bootstrap iterations. Results for the highest coherence

627 for each monkey should be interpreted carefully due to the extremely low number of error trials

628 for these conditions resulting from excellent behavioral performance. 

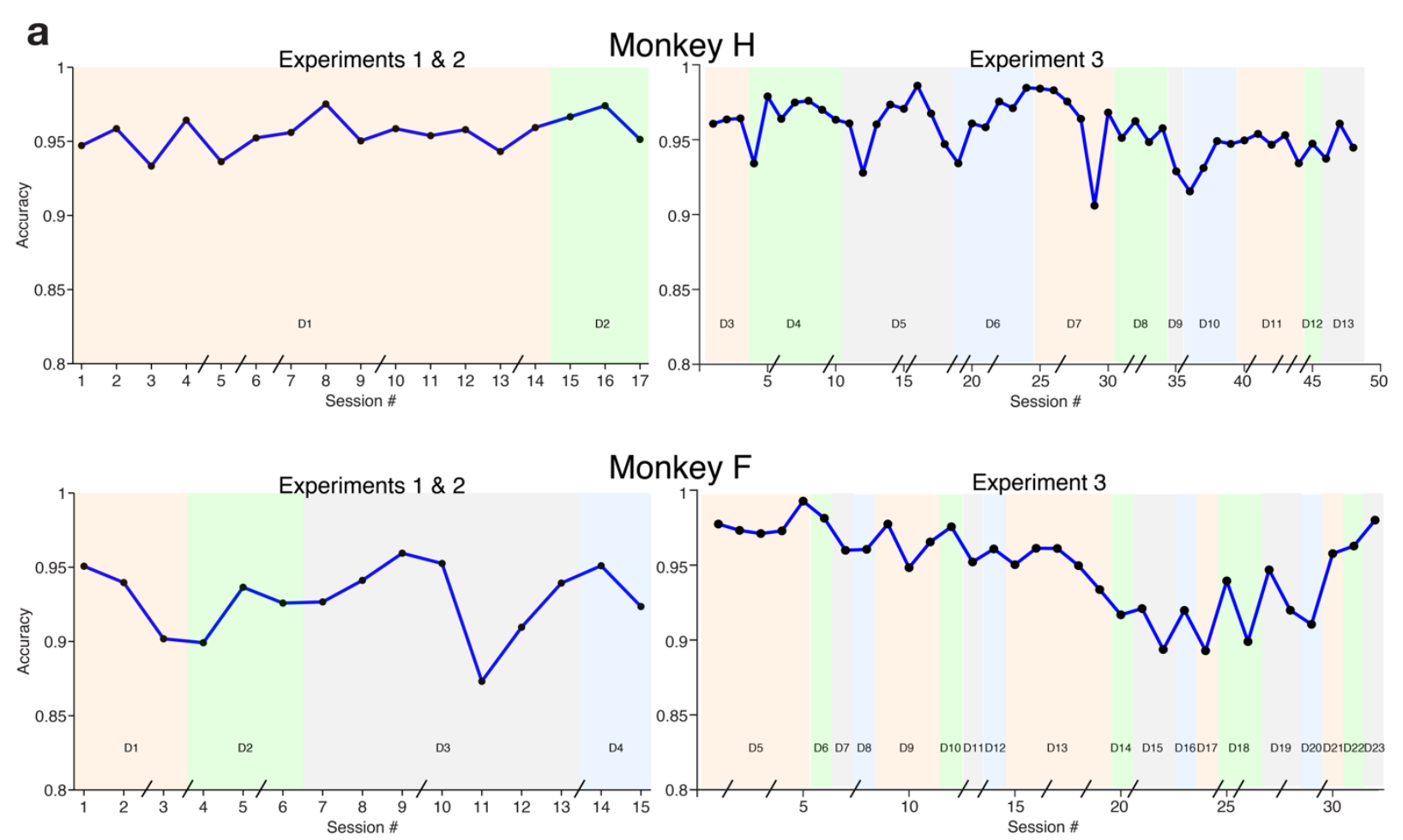

b
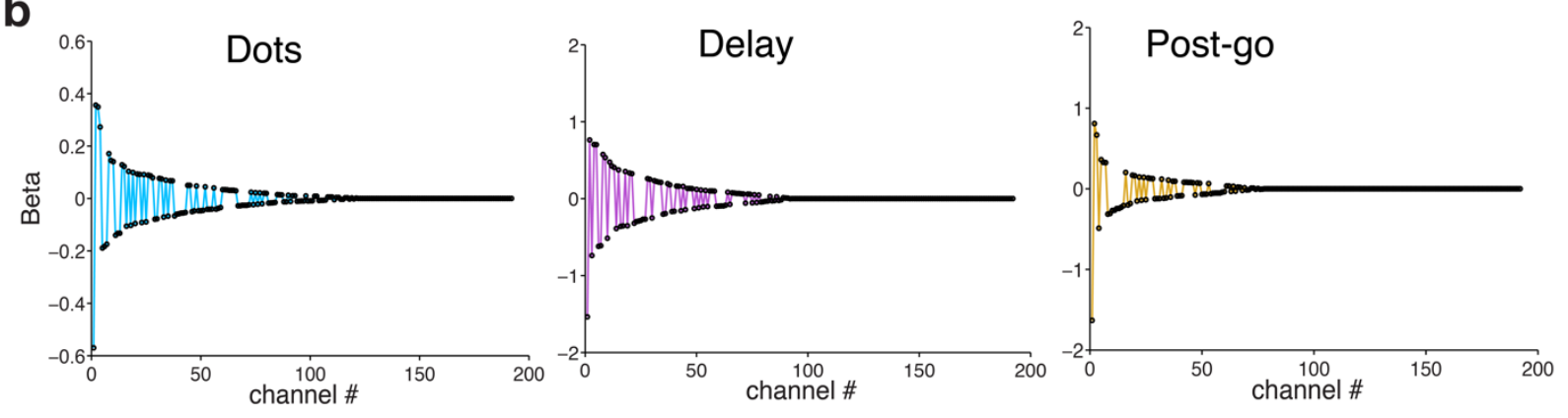

Extended Data Figure 5 - Real time decoding: performance reliability and decoder weights.

633 a) Decoding performance is stable across sessions. Average prediction accuracy during the second half of the stimulus presentation (600-1200 ms) across all sessions for monkey $\mathrm{H}$ (top

635 panels) and monkey F (bottom panels). D1-D23 denote different decoders (sets of beta weights)

636 used for the recorded sessions. For monkey H the same decoder (D1) was used for the first 14

637 sessions. The breaks on the x-axis correspond to sessions that occurred on non-consecutive days. 
b) Real time decoder beta weights. Beta weights during the dots period (left panel) ranked by absolute magnitude for an example decoder used in real time experiments. Channels with no or

640 little choice predictive activity during this period had their weights set to zero by using LASSO

641 regularization to prevent over fitting. Delay period and Post go cue Beta weights are shown in the 642 middle and right panels respectively.

643

644

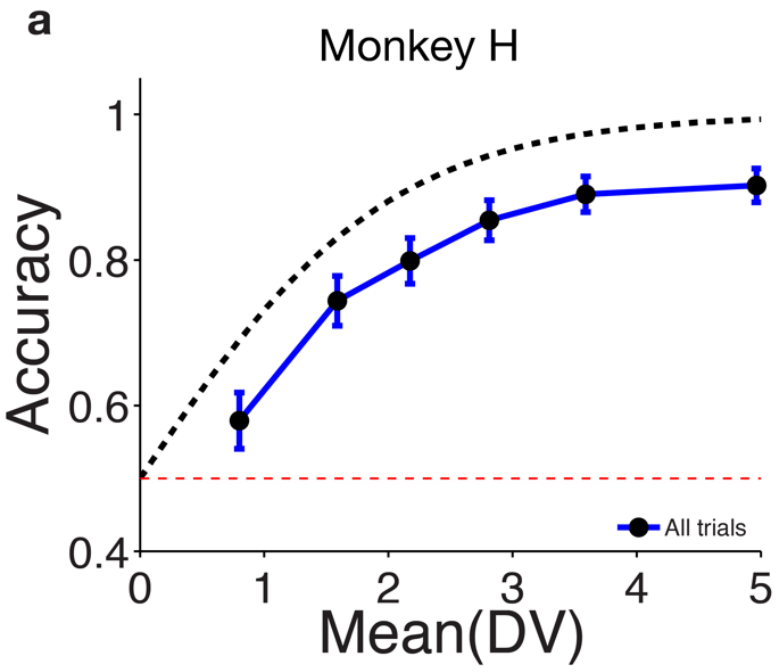

b

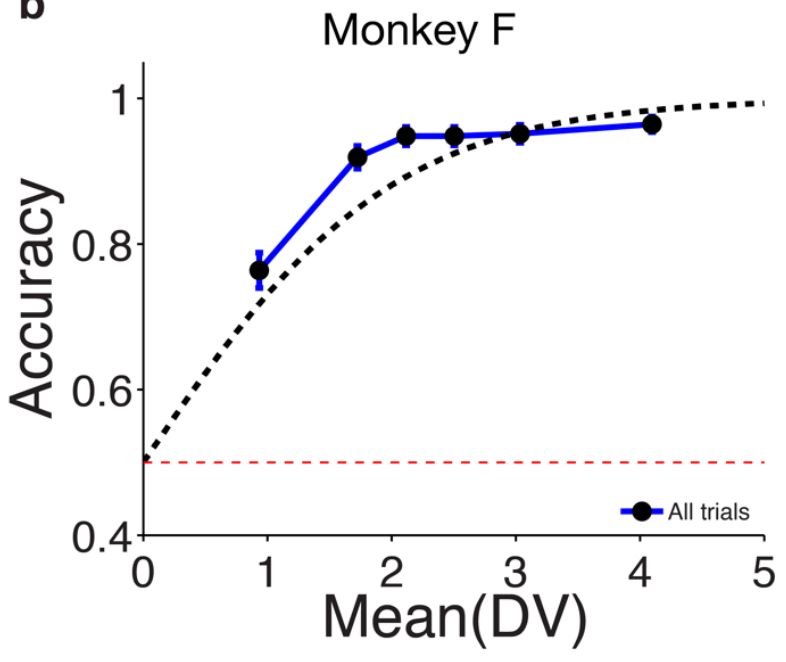
accuracy and median DV magnitude was calculated and plotted separately for each quantile (blue

651 line with black markers). Blue error bars show standard error of the mean for a binomial 652 distribution. Dashed black line shows predicted accuracy from log-odds equation and red dashed 653 line shows chance level. Data from 985 CoM trials from monkey $\mathrm{H}$. 


$$
\Delta \mathrm{DV}=\text { Within trial variability }
$$

a

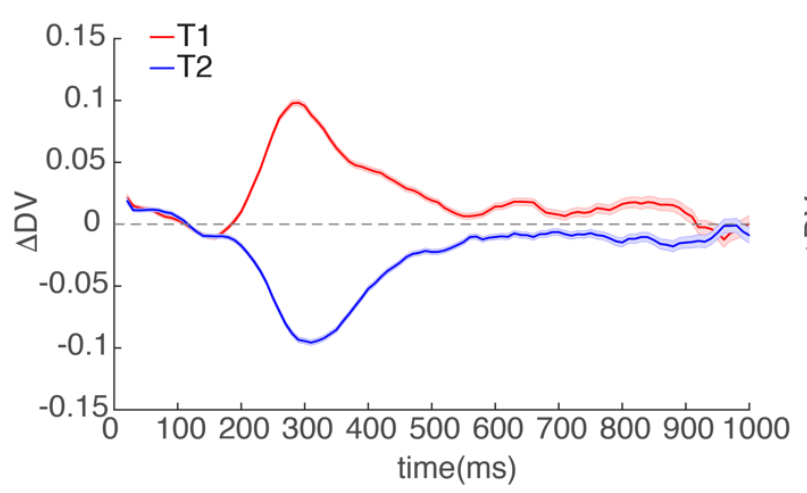

b

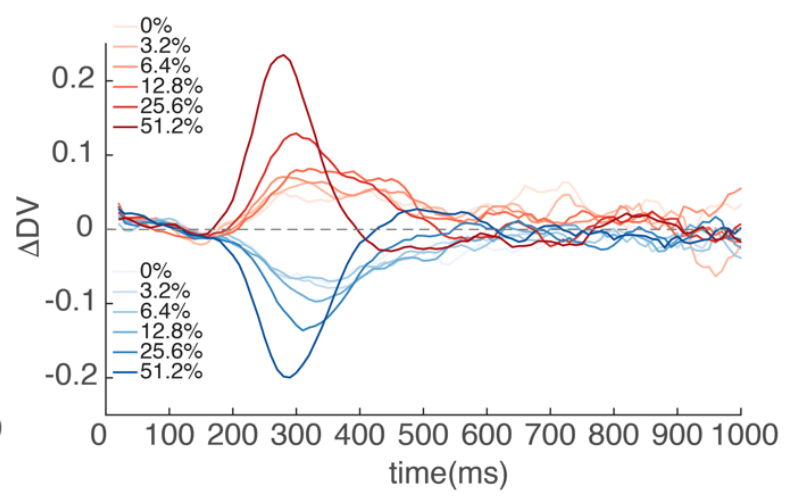
coherence. calculated for each trial as the difference between consecutive DV estimates spaced out by $10 \mathrm{~ms}$.

663 Traces show average DV derivative +/- s.e.m for right choices (red trace) and left choices (blue 664 trace). 
Monkey H Monkey F
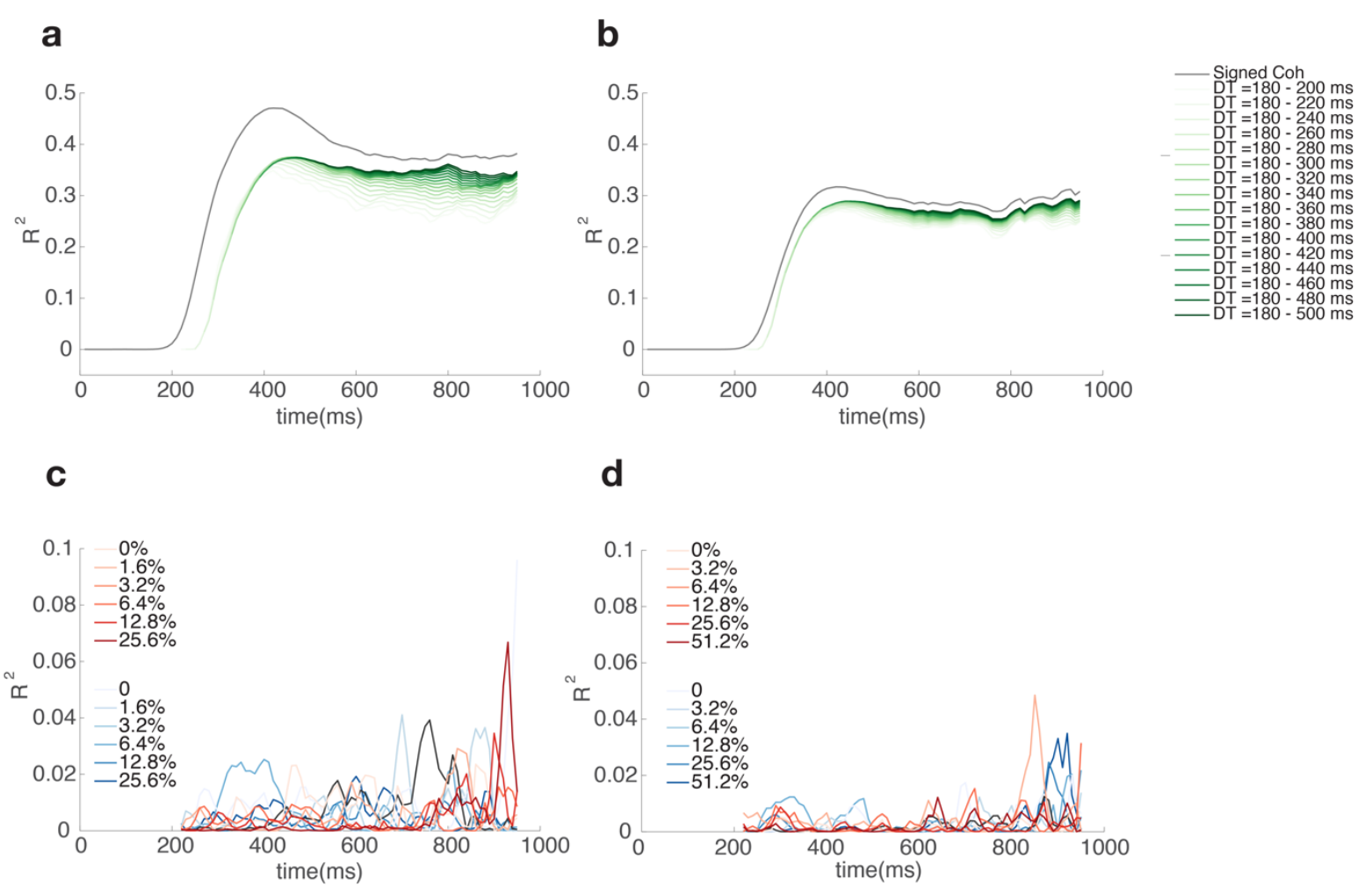

673 a) Correlation between stimulus motion energy and decision variable - monkey H. Proportion

674 of variance explained when regressing decision variable as a function of signed stimulus coherence

675 (grey trace) or motion energy (green traces). Each green trace corresponds to a separate regression

676 between DV and average motion energy between a timepoint in the past (from -200 ms up to -500

$677 \mathrm{~ms}$ ) and $-180 \mathrm{~ms}$ (the estimated neural response delay). Darker traces correspond to regressions in

678 which the motion energy was averaged for a longer time window. Across all coherence levels

679 motion energy and signed coherence explain a large fraction of DV variance.

680 b Correlation between stimulus motion energy and decision variable - monkey F. Same as a)

681 for monkey F. 


\section{c) Correlation between stimulus motion energy and decision variable within each level of}

683 signed coherence level - monkey H. Proportion of variance explained when regressing DV for

684 each time point and within each level of signed coherence as a function of the motion energy

685 preceding it by $180 \mathrm{~ms}$ (the estimated neural response delay). Within each level of signed

686 coherence, the DV fluctuations are not predicted by the motion energy traces

687 d) Correlation between stimulus motion energy and decision variable within each level of

688 signed coherence level - monkey F. Same as c) for monkey F.

690 Methods

694 Our experiments were performed on two adult male macaque monkeys (Macaca mulatta) trained

695 to perform a direction discrimination task with reaching movements of the arm as operant 696 responses. These were the same subjects used in our previous study ${ }^{10}$, but with new experiments.

697 All training, surgery, and recording procedures conformed to the National Institutes of Health

698 Guide for the Care and Use of Laboratory Animals and were approved by Stanford University 699 Animal Care and Use Committee.

$701 \quad$ Apparatus

703 Monkeys sat in a custom-made primate chair (Stanford Machine Shop) in front of a video 704 touchscreen, with their heads restrained using a surgical implant. The front plate of the chair could 
705 be opened, allowing the subjects to reach the touchscreen with the arm contralateral to the

706 implanted hemisphere. The ispsilateral arm was gently restrained using a delrin tube and a cloth

707 sling. Stimuli were shown on the video touchscreen (ELO Touchsystems 1939L), which was

708 positioned approximately $35.5 \mathrm{~cm}$ away from the monkeys' heads and allowed hand position to be

709 tracked at $75 \mathrm{~Hz}$. Eye position was continuously tracked with an infrared eye tracker at $1 \mathrm{kHz}$

710 (EyeLink 1000, SR Research, Canada).

711

\section{Motion Discrimination Task}

714 The task employed is a variation of the classical random dots motion discrimination task, in which

715 the subject uses an arm movement as the operant response ${ }^{10}$ (Fig. 1a). We used a variable duration

716 version of this task in which the duration of the stimulus presentation varied from trial to trial.

717 There were two types of trials in our experiments: open loop, in which the stimulus duration was

718 determined by the experimenter at the beginning of the trial and closed loop, in which the duration

719 was contingent on a specific pattern of neural activity detected in real time (see Experiments 1-3).

720 The subject was never cued on what type of trial it was on. For open-loop trials stimulus duration

721 ranged from 500-1200 ms (median $670 \mathrm{ms)}$ and was randomly chosen on each trial by sampling

722 an exponential distribution. For closed-loop trials the possible values for duration ranged between

$723 \quad 250-1200 \mathrm{~ms}$ and were determined on each trial either by the timepoint at which the termination

724 conditions were met or a predetermined random duration sampled from the open loop distribution,

725 whichever came first. All trials started with the onset of a fixation point (FP; 1.5 degree diameter)

726 on the video touchscreen (Fig. 1a). To initiate the task, the monkey was required to maintain both

727 eye and hand fixation within $+/-3$ degrees of the FP as long as it remained on the screen. 
728 Importantly, throughout the entire trial, the monkey was required to always maintain direct hand

729 contact with the screen, otherwise the trial would be aborted.

731 After $300 \mathrm{~ms}$ of fixation, two targets (1.5 degree diameter) appeared on opposite sides of the FP

732 (eccentricities between 10 and 17 degrees). After a 500 ms delay the random dot stimulus was

733 presented for the durations mentioned above, after which it was removed from the screen. The

734 monkey was asked to report the net direction of motion ( 0 or 180 degrees) by reaching to the target

735 in the corresponding direction. The difficulty of the task was adjusted by changing the fraction of

736 dots moving coherently in one direction (motion strength). After stimulus offset the monkey either

737 entered a delay period during which it was required to withhold his response for 400-900 ms (on

$73830 \%$ of the open-loop trials) or was immediately presented the go cue (on $70 \%$ of the open-loop

739 trials and all closed-loop trials). The go cue was then signaled by the offset of the FP at which

740 point the monkey was free to gaze anywhere and report his decision with his arm by reaching one

741 of the two targets. Although gaze was monitored, reward acquisition depended solely on reaching

742 to the correct target. Finally, for a response to be considered valid, the monkey was required to

743 hold its hand position within +/- 4 degrees of the center of the target for $200 \mathrm{~ms}$. The monkey was

744 then rewarded with a drop of juice for correct choices and given a timeout (2-4 seconds) for

745 incorrect ones. Zero coherence trials were rewarded randomly with a probability of 0.5 since there

746 was no correct response on these trials. The motion discrimination task was run on an Apple Mac

747 Pro running Mac OS.

\section{Random dots stimuli}


751 The stimuli used in our psychophysical experiment were random dot kinematograms (RDK)

752 generated using MATLAB and Psychophysics Toolbox. The details for generating the random

753 dots stimuli have been described previously ${ }^{10}$. However, to allow for closed loop experiments 1

754 and 2 (see below) we introduced a modification to be able to terminate the dot presentations early

755 if needed. The stimulus code was designed to precompute a sequence of kinematograms that

756 contain both random and moving dots. The sequence was then presented ballistically with no need

757 to continuously compute the content of each frame. Our modification allowed for DV values to be

758 received asynchronously from the real-time decoder and evaluated during the dots presentation. If

759 the DV criteria defined by the particular experiment were met, the dot presentation could then be

760 terminated without the remaining frames being shown. For the experiment in which an additional

761 pulse of motion energy was injected (closed loop experiment 3, see below), we arranged for two

762 sequences of kinematograms to be precomputed before presentation: one without the pulse, the

763 other for the $200 \mathrm{~ms}$ pulse itself. Contingent on the evolution of DV values, the stimulus could

764 then be rapidly switched from the standard sequence to the pulse sequence.

766 For both monkeys, the motion strength could take one of 6 possible values within a set, but the

767 sets were slightly different between subjects: $[0 \%, 1.6 \%, 3.2 \%, 6.4 \%, 12.8 \%, 25.6 \%]$ for monkey

$768 \mathrm{H}$ and $[0 \%, 3.2 \%, 6.4 \%, 12.8 \%, 25.6 \%, 51.2 \%]$ for monkey $\mathrm{F}$. The top coherence $(51.2 \%)$ was

769 dropped and a very low coherence (1.6\%) was introduced for monkey $\mathrm{H}$, due to its superior

770 discrimination ability. The direction and coherence of the motion were randomly assigned on each

771 trial by sampling from a uniform distribution with replacement. For zero-coherence stimuli all dots

772 were displaced randomly but, due to the stochasticity of that process, one obtains non-zero net

773 motion toward the targets over a small number of frames. 


\section{Behavioral Training}

776

777 Both monkeys had been extensively trained on fixed and variable duration versions of the motion

778 discrimination task using an arm reach movement as the operant response prior to the current

779 study $^{10}$. A few training sessions (all open-loop trials) were used to get the subject accustomed to

780 the new task timing (0.5-1.2 s stimuli and no delay on $70 \%$ of the trials). Real time decoding

781 sessions only started when psychophysical performance was stable.

782

\section{Behavioral Analysis}

785 Psychophysical performance was assessed in two ways: by describing the percentage of correct

786 choices as a function of (unsigned) stimulus coherence and by describing the percentage of

787 rightward choices as a function of signed stimulus coherence.

789 The percentage of correct choices as a function of motion strength (stimulus coherence) was fit by

790 a cumulative Weibull distribution function:

$$
P_{\text {correct }}(c)=1-0.5 \times e^{\left(-\frac{c}{\alpha}\right)^{\beta}}
$$

794 where $P_{\text {correct }}$ is probability correct, $c$ is motion strength, $\alpha$ is the psychophysical threshold (the

795 value of $c$ that corresponds to $\sim 82 \%$ correct responses), and $\beta$ is a parameter that controls the shape

796 of the function, especially its steepness. 
798 The proportion of rightward choices, $P_{\text {right }}$, as a function of motion strength and direction was fit

799 by a logistic regression:

$$
P_{\text {right }}(c)=\frac{1}{1+e^{-\beta_{1} \times\left(\beta_{0}+c\right)}}
$$

802

803 where $c$ is motion strength, $\beta_{1}$ is the slope parameter and $-\beta_{0}$ is the motion strength corresponding

804 to the indifference point. This value was used to assess the monkey's behavioral bias on each 805 session.

\section{Electrophysiological recordings}

809 Two multielectrode arrays (Blackrock Microsystems, Utah) with 96 electrodes each (1mm long

810 platinum-iridium electrodes, $0.4 \mathrm{~mm}$ spacing, impedance average of approximately $400 \mathrm{KOhm}$ )

811 were implanted in primary motor and dorsal premotor cortex of each monkey (Figure 1c). The

812 methods for determining the array placement were described in our previous study ${ }^{10}$. For monkey

$813 \mathrm{~F}$, the M1 array became unusable between the end of the previous study and the start of the current

814 study. Due to lack of neural signal from the M1 array, only the PMd array was used for this animal.

815 Continuous neural data were acquired and saved to disk from each channel (sampling rate $30 \mathrm{kHz}$ )

816 and thresholded at -4.5 RMS using the Cerebus recording system (Blackrock Microsystems, Utah)

817 and two separate PCs (one for each array) running Windows 8. Waveforms corresponding to

818 threshold crossings were not sorted and each channel could contain one or more unit(s). Sorting

819 waveforms would require a significant lead-up time before the beginning of the experiment and 
820 could negatively affect the ability to combine data and use decoders across days (see below,

821 Decoder training). Since units were not isolated within each channel our resulting units were most

822 likely multi-unit clusters. Any extremely noisy channels were deactivated at the beginning of a

823 session, and all other channels were used in this study. Using only multi-units yielded comparable

824 prediction accuracy (Extended Data Figure 3 ) to our previous study ${ }^{10}$ in which both single and

825 multi-unit data was used.

826

827 Datasets

828

829 Data were collected in two sets of experiments. In the first set of experiments we performed Closed

830 Loop Experiments 1 and 2 (see below). For this set, for each monkey we analyzed all datasets that

831 met two behavioral inclusion criteria: 1) over 500 trials and 2) a behavioral bias $\left(\left|\beta_{0}\right|\right)$ under $4 \%$,

832 as determined by a logistic regression fit (see above). These criteria were imposed to ensure that

833 we have a sizeable number of trials per condition ( 6 coherence $\mathrm{x} 2$ directions $=12$ conditions $)$ and

834 that the behavior of the monkey is virtually unbiased, such that both neural and behavioral results

835 are more easily interpretable. These criteria resulted in a selection of 17/15 sessions for a total of

$83616468 / 15826$ trials for monkey $\mathrm{H} / \mathrm{F}$, respectively.

838 In the second set of experiments we performed Closed Loop Experiment 3. This set of experiments

839 was performed later, on separate sessions, but using the same two subjects, arrays and decoding

840 techniques as the first set. In this set of experiments, we analyzed all datasets with over 550 trials.

841 For all experiments in monkey H, PMD and M1 were recorded simultaneously. 


\section{Decoder training}

845 We chose to use a logistic regression classifier based on our previous results showing excellent

846 offline prediction accuracy in variable duration tasks ${ }^{10}$ and because of the direct probabilistic

847 interpretation of its output. Our decision variable (DV) was defined as the log odds ratio of

848 observing a particular behavioral choice $\left(T_{1}\right.$ or $\left.T_{2}\right)$ given the population response $\vec{r}$ :

$$
D V=\log \frac{P\left(T_{1} \mid \vec{r}\right)}{P\left(T_{2} \mid \vec{r}\right)}=\beta_{0}(t)+\sum_{i=1}^{n} \beta_{i}(t) \times r_{i}(t)
$$

851 Where $r_{i}(t)$ are the z-scored summed spike counts for each neuron and time window, $\beta_{0}$ is an

852 intercept term and $\beta_{i}(t)$ are the classifier weights (one for each unit and epoch). Data from all 853 electrodes with valid waveforms were combined.

855 For simplicity, we decided to use only 3 different decoders for an entire trial (Fig. 1a), instead of 856 a different one for each $50 \mathrm{~ms}$ time window in the trial ${ }^{10}$. We applied the first decoder from fixation

857 up to and including the dots period, the second for the delay period and the third for the post go 858 cue period. After extensive offline tests on a few sessions the precise epochs for classifier training 859 were defined as the following:

861 Dots epoch: $[150,1000]$ ms aligned to dots onset;

862 - Delay epoch: [250, 350] ms aligned to dots offset;

863 - Post-go cue epoch: [200,400] ms aligned to go cue;

865 LASSO regularization was applied to prevent over-fitting when calculating each set of beta 
866 weights. A Lambda parameter constraining the L1 norm of the Beta vectors was calculated

867 separately for each of the 3 decoders using 10-fold cross validation on the corresponding time

868 epochs listed above. For each decoder the Lambda value with minimum cross-validation error was

869 chosen. Extended Data Figure 5b shows beta weights for an example set of 3 decoders for monkey

$870 \mathrm{H}$ sorted by epoch and ranked by magnitude. Positive weights correspond to rightward preferring

871 channels while negative weights correspond to leftward preferring channels. LASSO

872 regularization sets weights of channels with little or no predictive activity to zero.

874 The linear classifier was determined offline using recently collected data (from real-time 875 experiments). All $50 \mathrm{~ms}$ samples of neural data during the selected period (above) for each epoch

876 were used to train the classifier. The classifier was trained on $90 \%$ of the trials and tested on $10 \%$

877 of the trials using 10-fold cross-validation. The weights from one of the cross-validation folds were

878 then used in the upcoming real-time experiments. Decisions to train new decoders were based on 879 experimenter judgment in attempts to optimize performance: if a substantial decrease in real-time 880 decoding performance and/or an increase in the DV offset at baseline was observed, a new 881 classifier was trained and used in the following session. New classifiers were typically used every 8825 sessions, but some proved to be stable over up to 14 sessions (Extended Data Figure 5a).

\section{Real time decoding}

886 An essential requirement to compute a real-time read-out of neural activity is the ability to 887 continuously and (nearly) instantaneously access and perform computations on the neural activity 888 being recorded. To accomplish this, the spikes for each channel were temporally smoothed using 
889 a causal half-Gaussian kernel with $50 \mathrm{~ms}$ standard deviation (to mitigate spurious Poisson

890 fluctuations) and summed for the most recent $50 \mathrm{~ms}$. These smooth spike counts were then stored

891 in a 192x1 (96x1 for monkey F) vector of neural activity and z-scored individually for each

892 channel, using previously calculated $\mu$ (mean) and $\sigma$ (standard deviation) vectors. Z-scoring neural

893 activity was crucial to ensure a reliable and stable real-time readout by preventing the highest firing

894 channels from dominating it. Finally, the z-scored neural activity was projected onto a previously

895 calculated linear decoder (a set of $\beta$ weights, one for each channel) to obtain our linear readout of

896 internal decision state: a real time decision variable (DV) ${ }^{1}$.

898 The value of the DV was updated every $10 \mathrm{~ms}$, reflecting the neural activity of the preceding 50

899 ms. Because we used a half-gaussian kernel, data preceding the $50 \mathrm{~ms}$ window also influenced our

900 DV estimate (with more recent spikes carrying more weight). $95 \%$ of the data contributing to the

901 spike counts was limited to the last $100 \mathrm{~ms}$ (i.e an additional $50 \mathrm{~ms}$ in the past to each $50 \mathrm{~ms}$

902 window). The DV value and its history on a single trial could then be used (if desired) to impose

903 conditions for termination of the random dots stimulus (experiments 1 and 2) or presentation of a

904 motion pulse (experiment 3), effectively closing the loop on the experiment.

906 While the $\beta$ weights were not updated online (during the course of one experiment), the $\mu$ and $\sigma$

907 vectors for each epoch were learned continuously during the course of the experiment, due to

908 changing recording conditions and signals from day to day. The $\mu$ and $\sigma$ vectors were initialized

909 at the beginning of the session using the values calculated offline when training the most recent

910 decoder. Once the session started, the initial $\mu$ and $\sigma$ vectors were blended with online calculated

911 values for the first 25 trials, using a blending factor $\alpha$ : 


$$
\left.\alpha_{j}=\max ((25-j) / 25,0)\right), \text { where } j \text { is the trial number. }
$$

914

915 For trial $j$, sample number $\mathrm{t}$ and for a given epoch in trial, the $\mu$ and $\sigma$ vectors were defined as a

916 weighted mixture between the initial values $\mu_{\text {initial }}\left(\right.$ epoch) and $\sigma_{\text {initial }}($ epoch) and the estimate of the

917 current session's values $\mu_{\text {current }}(\mathrm{t}$,epoch $)$ and $\sigma_{\text {current }}(\mathrm{t}$, epoch $)$ :

$$
\begin{aligned}
& \mu_{\text {blended }}(t, \text { epoch })=\alpha_{j} * \mu_{\text {initial }}(\text { epoch })+\left(1-\alpha_{j}\right) * \mu_{\text {current }}(t, \text { epoch }) \\
& \sigma_{\text {blended }}(t, \text { epoch })=\alpha_{j} * \sigma_{\text {initial }}(\text { epoch })+\left(1-\alpha_{j}\right) * \sigma_{\text {current }}(t, \text { epoch })
\end{aligned}
$$

920

921 After the first 25 trials $\alpha$ was set to zero which implies the $\mu$ and $\sigma$ vectors kept being continuously 922 updated throughout the session but were no longer blended with values from the previous days.

923 The update rule for $\mu_{\text {current }}(\mathrm{t}$, epoch) was:

$$
\mu_{\text {current }}(t, \text { epoch })=\frac{\left[\mu_{\text {current }}(t-1, \text { epoch })\right] * K+r}{K+1}
$$

$$
K=N_{\text {samples }}(t, \text { epoch })
$$

927 of spike count vectors obtained so far for this particular epoch.

928 The update rule for $\sigma_{\text {current }}(\mathrm{t}$, epoch) was:

$$
\sigma_{\text {current }}(t, \text { epoch })=\sqrt{\frac{K-1}{K} * \sigma_{\text {current }}^{2}(t-1, \text { epoch })+\frac{1}{K} *\left(r-\mu_{\text {current }}(t, \text { epoch })\right)^{2}}
$$

930 After updating the $\mu_{\text {current }}(\mathrm{t}$, epoch $)$ and $\sigma_{\text {current }}(\mathrm{t}$, epoch $)$ vectors, the number of

931 samples for the corresponding epoch was also updated: 


$$
N_{\text {samples }}(t, \text { epoch })=N_{\text {samples }}(t-1, \text { epoch })+1
$$

935 Importantly, even though we had only 3 different decoders (Fig. 1b) we effectively used 5 different 936 epochs: Fixation, Targets, Dots, Delay and Post Go-Cue. The Dots decoder was also used in the

937 Fixation and Targets epochs but because average firing rates are different between these, different $938 \mu$ and $\sigma$ vectors had to be used. Every $50 \mathrm{~ms}$ sample of neural data for a given epoch was used to 939 update the corresponding $\mu$ and $\sigma$ vectors as described above. We let the $\mu$ and $\sigma$ vectors converge

940 for $\sim 200-300$ trials, in the beginning of each experimental session, before starting any closed loop

941 experiments. One way to check for this convergence was to monitor the DV offset: the average

942 DV value for the first $150 \mathrm{~ms}$ of the Dots epoch. Since we verified through offline analyses that 943 no systematic pre-planning activity towards one of the two targets was present in PMd or M1

944 during this time window, we expected the DV offset to be on average $\sim 0$.

946 Using a single decoder for an entire epoch was far more efficient to implement than using a

947 different decoder for each time point (as it reduced the number of $\mu$ and $\sigma$ vectors that had to be 948 learned online) and as demonstrated in our previous study ${ }^{10}$ a single classifier for an entire epoch 949 was almost as predictive as multiple classifiers trained on different timepoints of the same epoch ${ }^{10}$.

950 Because choice modulation in $\mathrm{PMd} / \mathrm{M} 1$ changes dramatically around the peri-movement period a 951 single decoder for an entire trial was not feasible.

953 In the end, our method yielded a reliable real-time decision state read out and required only $\sim 18 \%$ $954(15 \%)$ of trials in a session for calculating the values of $\mu$ and $\sigma$ for monkey $H(F)$, leaving the 955 remainder available for imposing neurally contingent conditions in closed loop. The real time 
956 decoder was run on two separate PCs (server and client) using the Simulink Real-Time/xPC

957 platform (Mathworks, Massachussetts).

958

\section{Closed Loop Experiments}

961 Experiment 1: Virtual Boundaries

962

963 On each trial we set a virtual threshold, or boundary (B), for the magnitude of the DV during the

964 dots epoch. If the DV on the current trial reached $\mathrm{B}$ or $-\mathrm{B} \pm$ tolerance, the dots presentation was

965 terminated and the monkey asked to report its decision. If the bound was not reached on a given

966 trial, stimulus presentation continued to a preset duration for that trial which was randomly

967 sampled from an exponential distribution ranging from 500-1200 ms. Closed loop trials for which

968 the boundary was not reached were effectively indistinguishable from open loop trials.

969

970 Typically, 5 values for boundaries spanning 0.5 to 5 (DV units) were used every session and one

971 of them was randomly assigned on each trial (uniform distribution). The tolerance used was \pm

972 0.25 DV units. We imposed a minimum duration for all trials to avoid spurious bound crossings,

973 which could be problematic for low bound values in particular. In all sessions the minimum

974 duration was $250 \mathrm{~ms}$, a conservative estimate of the latency for choice related signals driven by

975 the visual stimulus to appear in PMd and M1.

976

977 After the minimum stimulus duration was reached, the DV was assessed every 10 ms to determine 978 whether it fell within $\pm 0.25 \mathrm{DV}$ units of the bound chosen for the current trial $(\mathrm{B}$ or $-\mathrm{B})$. If so, the 
979 stimulus was terminated within $34 \mathrm{~ms}$ of the boundary being met (see Estimated latency for real

980 time closed loop setup). If the bound for the particular trial was not reached, the presentation

981 continued up to the maximum stimulus duration selected for that trial which had been obtained by

982 randomly sampling from an exponential distribution: 500-1200 ms (median $670 \mathrm{ms).}$

983

984 Finally, closed loop trials were randomly interleaved with open loop trials in which no DV-

985 dependent termination condition was imposed. The motivation for interleaving closed loop and

986 open loop trials was to make it extremely hard for the monkey to learn that accelerating the

987 dynamics of choice related signals ${ }^{10}$ (potentially by recruiting more choice related neurons or

988 increasing their modulation) and thus hitting bounds sooner could potentially increase its reward

989 rate. Not accounting for this possibility could lead to an undesirable change in the monkey's

990 strategy during the course of the closed loop experiments, which could become problematic when

991 combining data across days.

992

993 Experiment 2: CoM detection

994

995 Under our logistic regression framework, the signature of a putative CoM is a sign change of the

996 decision variable. Since these sign changes could happen at any time during the trial, capturing

997 them required not only monitoring the most recent state of the DV, but its history throughout the

998 trial. Because there was noise in our DV estimation and DVs usually started close to 0 at the

999 beginning of the trial we imposed selection criteria to detect likely CoMs based on the neural data.

1000 A necessary feature for all potential CoMs was a zero crossing in the sign of the DV: change of

1001 DV sign from negative to positive reflected a change in the likelihood of a rightward decision from 
1002 less than $50 \%$ to greater than $50 \%$, and vice versa for the opposite change in sign. To eliminate

1003 zero crossings resulting solely from measurement noise, we imposed four additional criteria:

1004

1005 - Minimum DV value after zero crossing;

1006 - Minimum DV value with opposite sign before zero crossing;

1007 - Minimum duration of DV sign stability after zero crossing;

1008 - Minimum duration of DV sign stability before zero crossing;

1009

1010 The minimum DV values before and after zero crossing were symmetrical for most sessions, as

1011 were the periods of minimum duration of DV sign stability (negative or positive values for all time

1012 points). If a zero crossing was detected and all four criteria were met, the stimulus presentation

1013 was interrupted and the animal was virtually immediately (within $34 \mathrm{~ms}$ or less, see Estimated

1014 latency for real time closed loop setup) prompted to report a decision. The exact parameters used

1015 for each session can be found in Supplementary Information Table 3.

1016

1017 By sweeping the parameter space we could test zero crossings that differed in magnitude and

1018 stability. Analogously to the virtual boundary experiment, if the minimums were not met and a

1019 CoM thus not detected, the stimulus presentation continued uninterrupted for a random duration

1020 ranging from 500-1200 ms, selected prior to the start of the trial. A minimum stimulus duration of

$1021250 \mathrm{~ms}$ was also in place.

1022

1023 Because putative CoMs are quite rare ${ }^{1}$, in the first set of experiments we devoted $70 \%$ of the closed

1024 loop trials to detect them leaving the remaining 30\% as virtual boundary trials. The exact fraction 
1025 of trials with CoM depends dramatically on how we parameterize them. The longer the minimum

1026 periods of consistent sign and the higher the minimum DV value in the initial commitment stage,

1027 the rarer they become. Running both experiments on the same sessions ensured that the mapping

1028 from DV to choice likelihood was held during the CoM experiments and provided the most faithful

1029 indirect validation of initial commitment we could obtain.

1030

Experiment 3: Motion pulse perturbation

1033 In this experiment, motion pulses were introduced on some trials with motion coherences near or

1034 below psychophysical threshold. No motion pulses were presented for suprathreshold coherences

1035 based on the results of a pilot experiment (not shown) in which pulses presented at suprathreshold

1036 coherences were more perceptually salient and led to changes in the animals' strategy. As in

1037 Experiment 1, on each trial we set a virtual boundary (B) for the magnitude of the DV during the

1038 dots epoch. In this experiment, $100 \%$ of trials with dots coherence at or near psychophysical

1039 threshold were treated as closed loop trials (this corresponds to trials with maximum unsigned

1040 coherence of $6.4 \%$ for monkey $\mathrm{H}$ and $12.8 \%$ for monkey $\mathrm{F}$; psychophysical thresholds were

1041 measured using the Weibull function described above in "Behavioral Analysis"). Low-coherence

1042 trials in which the boundary was not reached (per the criteria below) and trials with suprathreshold

1043 dots coherences were all effectively open loop.

1045 If the DV on a closed loop trial reached $\mathrm{B}$ or $-\mathrm{B} \pm$ tolerance $( \pm 0.25 \mathrm{DV}$ units), after a minimum

1046 stimulus duration of $50 \mathrm{~ms}$, a $200-\mathrm{ms}$ motion pulse was presented, followed immediately by

1047 termination of the visual stimulus and presentation of the cue for the monkey to report its decision. 
1048 If not, dots presentation continued for a pre-assigned duration drawn randomly from an 1049 exponential distribution of 500-1200 ms. Four integer values for boundaries (spanning 1 to 4 DV 1050 units) were used every session, and one of them was randomly assigned on each trial (uniform 1051 distribution).

1053 Motion pulses were 200-ms periods of additional dots stimulus presentation with small additive 1054 average coherence $( \pm 2 \%$ or $4.5 \%$ from the initial dots coherence on the same trial for monkey $\mathrm{H}$ 1055 and F, respectively, where positive coherence values indicate rightward motion); thus pulses 1056 effectively randomly added either a small amount of rightward or leftward motion evidence to the 1057 stimulus. Pulse strength was calibrated in pilot experiments, in which we converged upon 1058 coherence shifts that slightly but significantly biased each animal's behavior, without being overtly 1059 perceptually salient (biases were measured using the logistic regression on rightward choice 1060 described above in "Behavioral Analysis"). Animals were rewarded for correct reaches in the 1061 direction of the coherence of the initial dots stimulus (randomly assigned on $0 \%$ coherence trials), 1062 regardless of the pulse direction.

1063

1064 Estimated latency for real time closed loop setup

1066 To validate our setup, we measured the latency between a neural condition being met and the 1067 corresponding task change being implemented. We tested this latency by generating simulated DV 1068 steps in the same model used to detect when DV triggering conditions were met in the real 1069 experiments. We used these simulated steps to trigger the onset of a bright light on the touchscreen 1070 in front of a photodetector, again within the same code used to run the task and generate the stimuli 
1071 in the real experiments. We then passed both the simulated DV and the photodetector output

1072 signals into an oscilloscope, triggered the display on the "DV" steps, and manually measured the

1073 delay to onset of the bright dot. Almost all measured delays were within 2 frames, or 26 ms.

1074

1075 Estimated trial count savings for real time closed loop setup

1076

1077 The real time setup allowed for extremely precise experimental control over which DV values or

1078 DV history to use to trigger a modification in the task (stimulus termination or pulse). However, it

1079 could be argued that given enough data, similar trials would have been captured simply by either

1080 terminating the stimulus (as in experiments 1 and 2) or presenting the pulse (as in experiment 3 )

1081 at a random point in the trial and then back sorting them offline (by DV value or history by after

1082 the data is collected).

1083

1084 To estimate how much more trial-count efficient it was to use our real time setup compared to

1085 offline back-sorting trials where the stimulus was presented for a random duration, we used the

1086 CoM experiment as a case study given how rare change of mind events are.

1088 For simplicity, we focused on sessions 1, 2 and 3 from Monkey F, which all have the same (and

1089 intermediate) CoM requirements (Supplementary Information Table 3). We started by calculating

1090 the yield from the real time experiment in closed loop as the ratio between detected CoM trials and

1091 trials in which CoMs were checked (i.e. all closed loop trials in which the stimulus could be

1092 terminated if the conditions dictated by the CoM parameters were met, Supplementary Information

1093 Table 3): 


$$
\text { Yield }_{C L}=\# \text { CoMs detected } / \# \text { trials CoMs checked }=11.91 \%
$$

1096 To calculate the yield for offline back-sorting trials we used the open loop trials in the same

1097 sessions, which were terminated after a random stimulus duration. Importantly, the stimulus

1098 duration on these open loop trials was sampled from the same distribution as for the closed loop

1099 trials in which CoMs were checked, which allows for a fair yield comparison. We calculated the

1100 yield from offline back-sorting as the ratio between the number of trials that would have met all

1101 the criteria for CoMs for the same session and the total number of open loop trials:

$$
\text { Yield }_{O L}=\# \text { Valid putative CoMs /\#Open loop trials }=1.85 \%
$$

1105 Since the goal would be to probe the new choice preference shortly after the zero crossing (putative

1106 change of mind), not many hundreds of ms later, we only considered CoM trials that were (closed

1107 loop) or that would have been (open loop) terminated within $150 \mathrm{~ms}$ of the zero crossing. This 1108 cutoff value corresponded to the $82^{\text {nd }}$ percentile of post zero crossing durations for the closed loop 1109 trials analysed in these sessions.

1111 In this analysis Yield ${ }_{\mathrm{CL}}$ was 6.43 times higher than Yield $\mathrm{OL}_{\mathrm{L}}$. This result implies that had we not 1112 used a real time setup in closed loop we would have had to collect 6.43 times the number of trials 1113 (and thus sessions) to obtain the same number of events. This would in turn mean collecting 1114 around 100 sessions/monkey just for experiments 1 and 2 (assuming the same 30\%/70\% trial split 1115 used in the real time experiments), rendering this experiment practically unfeasible. 


\section{Neural Data Analysis}

1119 DV variability

1121 Within trial variability was computed by first calculating the difference between consecutive DV

1122 values (estimated every $10 \mathrm{~ms}$ ) for every trial in the datasets collected for experiments 1 and 2

1123 (open and closed loop). This step yielded a $\Delta \mathrm{DV}$ trace for each trial aligned to dots onset. For each

1124 trial these traces were computed only up to the offset of the stimulus and did not include any delay

1125 or post go-cue DV data. The $\Delta \mathrm{DV}$ traces were then sorted and averaged for each choice (Fig. 5a,

1126 Extended Data Fig.7a) or each signed coherence level (Fig. 5b, Extended Data Fig.7b). Longer

1127 trials are increasingly rare due to the shape of our stimulus duration distribution but this asymmetry

1128 does not influence the interpretation of the time course of average $\Delta \mathrm{DV}$ as this metric only captures

1129 within trial variability and not across trial variance.

1131 DV and motion energy correlation

1133 Motion energy (ME) was calculated for each trial in the datasets collected for experiments 1 and

11342 (open and closed loop) by convolving the positions of the dots in the stimulus with spatio-

1135 temporal filters as previously described ${ }^{8}$. The ME trace obtained for each trial captures the strength

1136 of the stimulus at every timepoint during the stimulus presentation. To evaluate the effect of motion

1137 energy on DV we performed a linear regression of single trial DV traces on single trial ME traces.

1138 From experiments 1 and 3 we determined that due to neural latencies a stimulus fluctuation can 1139 only have effect on the decoded DV $\sim 180 \mathrm{~ms}$ later. For this reason, the regression was always 
1140 performed between DV(t) and ME(t-180ms) or earlier. For Extended Data Fig. 8a-b each green

1141 trace corresponds to a different way to estimate the motion energy that might affect DV at time t.

1142 For the lightest trace and for every timepoint $\mathrm{t}$ ME was averaged between ( $\mathrm{t}-180 \mathrm{~ms})$ and (t-200ms)

1143 for every trial and used to regress against DV(t). A separate regression was performed for each

1144 timepoint and the resulting variance explained was plotted. The same process then was repeated

1145 for every other green trace by progressively increasing the averaging window for ME in $20 \mathrm{~ms}$

1146 increments from (t-180, t- 200) ms to (t-180, t- 500) ms. As a control the DV was also regressed

1147 against signed coherence for each trial (Extended Data Fig. 8a-b grey traces). This analysis was

1148 used to assess how much of the DV variance across coherences is explained by motion energy or

1149 signed coherence as a function of time. To assess how much DV variance within each coherence

1150 level could be explained by the motion energy of the stimulus we first sorted the DV traces for

1151 each signed coherence level. For each signed coherence level and each timepoint we regressed

$1152 \mathrm{DV}(\mathrm{t})$ against $\mathrm{ME}(\mathrm{t}-180 \mathrm{~ms})$ for the corresponding trials and calculated the variance explained

1153 (Extended Data Fig. 8c-d).

\section{CoM regularities}

1157 To test whether the effects of coherence on the number of CoMs were statistically significant we

1158 used a bootstrap method to generate 1000 distributions of CoM events with the corresponding

1159 coherences by sampling with replacement from the distribution of captured events for each subject

1160 separately. For each subject each distribution had the same number of observations as those

1161 captured in experiment 2: 985 for monkey $\mathrm{H}$ and 1727 for monkey F. For each randomly sampled

1162 distribution the number of CoMs for each coherence level was counted. The resulting counts were 
1163 then regressed against the coherence level they belonged to. CoM count was highly and negatively

1164 correlated with coherence for both subjects $(\mathrm{p}<0.001)$.

1165 To test whether the effect of directionality of CoMs (corrective vs erroneous) was statistically

1166 significant we followed a similar bootstrapping procedure and generated 1000 distributions of

1167 CoM events (excluding 0\% trials) with the corresponding directionality. For each randomly

1168 sampled distribution the number of CoMs for each direction was counted. The difference between

1169 the medians counts of corrective and erroneous CoMs was tested by performing a one-sided

1170 Wilcoxon rank sum test $(\mathrm{p}<0.001)$ testing the hypothesis than corrective counts were higher than

1171 erroneous counts.

1173 Pulse Effects

1175 To quantify the overall behavioral effect of the pulses, we performed the following logistic 1176 regression on the probability of a rightward choice:

$$
P_{\text {right }}(c)=\frac{1}{\left.1+e^{-\beta_{1} \times\left(\beta_{0}+\beta_{p u l s e}\right.}+c\right)},
$$

1180 where $c$ is motion strength, $\beta_{1}$ is the slope parameter, $D$ is the pulse direction, and $-\beta_{0}$ is the motion 1181 strength corresponding to the indifference point.

1183 To determine the effect of the pulse on the evolving DV, we first estimated the minimum latency

1184 for the visual stimulus information to influence the DV by calculating the first time of significant

1185 divergence of rightward vs. leftward DV traces during dots presentation on open-loop trials with 
1186 stimuli of maximal motion strength $( \pm 25.6 \%, 51.2 \%$ coherence for monkey $\mathrm{H}, \mathrm{F})$, assessed using

1187 a two-sample t-test with correction for a false discovery rate of $0.05^{34}$. We refer to this estimate as

1188 the "evidence representation latency" (ERL; $170 \mathrm{~ms}, 180 \mathrm{~ms}$ for monkey H, F). We then measured

1189 the evolution of the DV after pulse presentation by calculating the difference between the

1190 empirically observed DV at each time point $t$ and the DV at the "pulse evidence representation

1191 latency" (PERL, or time of pulse onset plus the ERL):

$$
\triangle D V(t)=D V(t)-D V_{P E R L}
$$

1195 To quantify the pulse effect on DV on single trials, we calculated the slope (using linear regression)

1196 of $\triangle \mathrm{DV}$ over the time period beginning with the PERL and ending at either the median go-RT for

1197 the animal or $150 \mathrm{~ms}$ prior to movement initiation on that trial, whichever came first, to minimize 1198 the contribution of directly movement-related activity to our analysis of the evolving choice ${ }^{10}$. We

1199 checked to ensure the results did not depend critically on these specific endpoint criteria by 1200 sweeping a range of endpoints for the $\triangle \mathrm{DV}$ calculation: every $10 \mathrm{~ms}$ from go cue onset to $150 \mathrm{~ms}$ 1201 after the go cue. The exact endpoint used did not affect the results of the following analyses 1202 (quantitative results held for all endpoints from the time of the go cue through $150 \mathrm{~ms}$ post-go-cue 1203 for monkey $\mathrm{H}$, and for endpoints beginning $120 \mathrm{~ms}$ post-go-cue for monkey $\mathrm{F}$ ).

1205 To quantify the neural pulse effect at each DV boundary (as in Fig. 5e), we fit the following linear 1206 regression on the $\Delta \mathrm{DV}$ slope (calculated as described in the previous paragraph) separately for 1207 each DV boundary: 


$$
\begin{aligned}
\Delta D V \text { slope } & =\beta_{0}^{B}+\beta_{\text {coh }}^{B} \times c+\beta_{\text {time }}^{B} \times \text { time }_{\text {pulse onset }}+\beta_{\text {pulse }}^{B} \times D \\
& +\beta_{\text {pulse } \times \text { time }}^{B} \times\left(\text { time }_{\text {pulse onset }} \times D\right),
\end{aligned}
$$

1212 where $B$ is the pulse-triggering DV boundary (unsigned), and compared $\AA_{\text {pulse values. (The full }}^{B}$

1213 resulting sets of regression coefficients fit to $\Delta \mathrm{DV}$ slope can be found in Supplementary

1214 Information Table 1.)

1215

1216 Similarly, to quantify the behavioral pulse effect at each DV boundary (as in Fig. 5f), we fit the

1217 following logistic regression on the probability of a rightward choice separately for each DV

1218 boundary:

$$
\begin{gathered}
P_{\text {right }}^{B}(c)=\frac{1}{1+e^{-\left(z^{B}\right)}}, \\
\text { where } z^{B}=\beta_{0}^{B}+\beta_{\text {coh }}^{B} \times c+\beta_{\text {time }}^{B} \times \text { time }_{\text {pulse onset }}+\beta_{\text {pulse }}^{B} \times D \\
+\beta_{\text {pulse } \times \text { time }}^{B} \times\left(\text { time }_{\text {pulse onset }} * D\right),
\end{gathered}
$$

1223 where $B$ is again the pulse-triggering DV boundary (unsigned), and compared $\beta_{\text {pulse }}^{B}$ values. (The

1224 full resulting sets of regression coefficients fit to choice can be found in Supplementary 1225 Information Table 2.)

\section{Data availability}

1229 All data and analyses generated during the current study are available from the corresponding 1230 authors upon reasonable request. 
1232 Code availability

1233

1234 The analysis code was developed in Matlab (Mathworks) and is available from the corresponding

1235 authors upon reasonable request.

1236

1237

1238

1239

1240

\section{References}

1242

12431 Kiani, R., Cueva, C. J., Reppas, J. B. \& Newsome, W. T. Dynamics of neural population

1244 responses in prefrontal cortex indicate changes of mind on single trials. Curr Biol 24,

1245 1542-1547, doi:10.1016/j.cub.2014.05.049 (2014).

12462 Kaufman, M. T., Churchland, M. M., Ryu, S. I. \& Shenoy, K. V. Vacillation, indecision 1247 and hesitation in moment-by-moment decoding of monkey motor cortex. Elife 4, e04677, 1248 doi:10.7554/eLife.04677 (2015).

12493 Bollimunta, A., Totten, D. \& Ditterich, J. Neural Dynamics of Choice: Single-Trial 1250 Analysis of Decision-Related Activity in Parietal Cortex. The Journal of Neuroscience $1251 \quad 32,12684(2012)$. 
12524 van den Berg, R. et al. A common mechanism underlies changes of mind about decisions 1253 and confidence. eLife 5, e12192, doi:10.7554/eLife.12192 (2016).

12545 Lemus, L. et al. Neural correlates of a postponed decision report. Proceedings of the $1255 \quad$ National Academy of Sciences 104, 17174, doi:10.1073/pnas.0707961104 (2007).

12566 Rich, E. L. \& Wallis, J. D. Decoding subjective decisions from orbitofrontal cortex. Nat 1257 Neurosci 19, 973-980, doi:10.1038/nn.4320 (2016).

12587 Resulaj, A., Kiani, R., Wolpert, D. M. \& Shadlen, M. N. Changes of mind in decision1259 making. Nature 461, 263, doi:10.1038/nature08275

1260 https://www.nature.com/articles/nature08275\#supplementary-information (2009).

12618 Kiani, R., Hanks, T. D. \& Shadlen, M. N. Bounded integration in parietal cortex

1262 underlies decisions even when viewing duration is dictated by the environment. $J$

1263 Neurosci 28, 3017-3029, doi:10.1523/JNEUROSCI.4761-07.2008 (2008).

12649 Britten, K. H., Shadlen, M. N., Newsome, W. T. \& Movshon, J. A. The analysis of visual 1265 motion: a comparison of neuronal and psychophysical performance. J. Neurosci. 12, $1266 \quad 4745-4765$ (1992).

$126710 \quad$ Peixoto, D. et al. Population dynamics of choice representation in dorsal premotor and 1268 primary motor cortex. bioRxiv (2018).

126911 Shadlen, M. N. \& Newsome, W. T. Neural Basis of a Perceptual Decision in the Parietal 1270 Cortex (Area LIP) of the Rhesus Monkey. J Neurophysiol 86, 1916-1936 (2001).

1271 Ratcliff, R. \& McKoon, G. The Diffusion Decision Model: Theory and Data for Two1272 Choice Decision Tasks. Neural Computation 20, 873-922, doi:10.1162/neco.2008.12-06$1273 \quad 420(2007)$. 
1274 Mazurek, M. E. A Role for Neural Integrators in Perceptual Decision Making. Cerebral $1275 \quad$ Cortex 13, 1257-1269, doi:10.1093/cercor/bhg097 (2003).

127614 Lo, C.-C. \& Wang, X.-J. Cortico-basal ganglia circuit mechanism for a decision threshold 1277 in reaction time tasks. Nat Neurosci 9, 956-963, 1278 doi:http://www.nature.com/neuro/journal/v9/n7/suppinfo/nn1722_S1.html (2006).

127915 Usher, M. \& McClelland, J. L. The time course of perceptual choice: The leaky, $1280 \quad$ competing accumulator model. Psychological Review 108, 550-592, doi:10.1037/0033295X.108.3.550(2001).

128216 Cisek, P., Puskas, G. A. \& El-Murr, S. Decisions in Changing Conditions: The Urgency1283 Gating Model. The Journal of Neuroscience 29, 11560 (2009).

128417 Huk, A. C. \& Shadlen, M. N. Neural Activity in Macaque Parietal Cortex Reflects 1285 Temporal Integration of Visual Motion Signals during Perceptual Decision Making. The $1286 \quad$ Journal of Neuroscience 25, 10420 (2005).

128718 Kiani, R. \& Shadlen, M. N. Representation of confidence associated with a decision by 1288 neurons in the parietal cortex. Science (New York, N.Y.) 324, 759-764, 1289 doi:10.1126/science.1169405 (2009).

129019 Smith, P. L. \& Ratcliff, R. Psychology and neurobiology of simple decisions. Trends in 1291 Neurosciences 27, 161-168, doi:10.1016/j.tins.2004.01.006 (2004).

129220 Ditterich, J. Evidence for time-variant decision making. European Journal of $1293 \quad$ Neuroscience 24, 3628-3641, doi:10.1111/j.1460-9568.2006.05221.x (2006).

129421 Hanks, T., Kiani, R. \& Shadlen, M. N. A neural mechanism of speed-accuracy tradeoff in 1295 macaque area LIP. eLife 3, e02260, doi:10.7554/eLife.02260 (2014). 
129622 Wong, K.-F., Huk, A., Shadlen, M. \& Wang, X.-J. Neural circuit dynamics underlying

1297 accumulation of time-varying evidence during perceptual decision making. Frontiers in

1298 Computational Neuroscience 1, 6 (2007).

129923 Inagaki, H. K., Fontolan, L., Romani, S. \& Svoboda, K. Discrete attractor dynamics

$1300 \quad$ underlies persistent activity in the frontal cortex. Nature 566, 212-217,

1301 doi:10.1038/s41586-019-0919-7 (2019).

130224 Seidemann, E., Meilijson, I., Abeles, M., Bergman, H. \& Vaadia, E. Simultaneously

1303 recorded single units in the frontal cortex go through sequences of discrete and stable

1304 states in monkeys performing a delayed localization task. The Journal of Neuroscience

$1305 \quad \mathbf{1 6}, 752(1996)$.

130625 Golub, M. D., Chase, S. M., Batista, A. P. \& Yu, B. M. Brain-computer interfaces for

1307 dissecting cognitive processes underlying sensorimotor control. Current Opinion in

1308 Neurobiology 37, 53-58, doi:https://doi.org/10.1016/j.conb.2015.12.005 (2016).

130926 Sussillo, D., Stavisky, S. D., Kao, J. C., Ryu, S. I. \& Shenoy, K. V. Making brain-

1310 machine interfaces robust to future neural variability. Nature Communications 7, 13749,

1311 doi:10.1038/ncomms13749

1312 https://www.nature.com/articles/ncomms13749\#supplementary-information (2016).

131327 Semedo, J. D., Zandvakili, A., Machens, C. K., Yu, B. M. \& Kohn, A. Cortical Areas

1314 Interact through a Communication Subspace. Neuron 102, 249-259.e244,

1315 doi:https://doi.org/10.1016/j.neuron.2019.01.026 (2019).

131628 Seideman, J. A., Stanford, T. R. \& Salinas, E. Saccade metrics reflect decision-making

1317 dynamics during urgent choices. Nature Communications 9, 2907, doi:10.1038/s41467-

1318 018-05319-w (2018). 
131929 Musall, S., Kaufman, M. T., Juavinett, A. L., Gluf, S. \& Churchland, A. K. Single-trial 1320 neural dynamics are dominated by richly varied movements. bioRxiv, 308288, 1321 doi:10.1101/308288 (2019).

$1322 \quad 30 \quad$ Aflalo, T. et al. Decoding motor imagery from the posterior parietal cortex of a 1323 tetraplegic human. Science 348, 906 (2015).

132431 Andersen, R. A., Hwang, E. J. \& Mulliken, G. H. Cognitive Neural Prosthetics. Annual 1325 Review of Psychology 61, 169-190, doi:10.1146/annurev.psych.093008.100503 (2009).

132632 Musallam, S., Corneil, B. D., Greger, B., Scherberger, H. \& Andersen, R. A. Cognitive 1327 Control Signals for Neural Prosthetics. Science 305, 258 (2004).

132833 Pesaran, B., Musallam, S. \& Andersen, R. A. Cognitive neural prosthetics. Current 1329 Biology 16, R77-R80, doi:10.1016/j.cub.2006.01.043 (2006).

133034 Benjamini, Y.\& Hochberg, Y. Controlling The False Discovery Rate - A Practical And 1331 Powerful Approach To Multiple Testing. Vol. 57 (1995).

\section{End notes}

1335 Supplementary Information is linked to the online version of the paper.

\section{Acknowledgments}

1339 We thank all other members of the Newsome and Shenoy labs at Stanford University for comments

1340 on the methods and results throughout the execution of the project. D.P. was supported by the 1341 Champalimaud Foundation, Portugal, and Howard Hughes Medical Institute. J.R.V. was supported 
1342 by Stanford MSTP NIH training grant 4T32GM007365. R.K. was supported by Simons

1343 Collaboration on the Global Brain grant 542997, Pew Scholarship in Biomedical Sciences,

1344 National Institutes of Health Award R01MH109180, and a McKnight Scholars Award. J.C.K. was

1345 supported by NSF graduate research fellowship. P.N. was supported by NIDCD award

1346 R01DC014034. C.C. was supported by K99NS092972 and 4R00NS092972-03 award from the

1347 NINDS and supported as a research specialist by the Howard Hughes Medical Institute. J.B. and

1348 S.F. were supported by the Howard Hughes Medical Institute. K.V.S. was supported by the

1349 following awards: NIH Director's Pioneer Award 8DP1HD075623, DARPA-BTO

1350 "NeuroFAST" award W911NF-14-2-0013, the Simons Foundation Collaboration on the Global

1351 Brain awards 325380 and 543045, and ONR award N000141812158. W.T.N. and K.V.S. were

1352 supported by the Howard Hughes Medical Institute.”

1353

1354 Author Contributions

1356 D.P., J.R.V., R.K., S.F., K.V.S. and W.T.N. designed the experiments. D.P., J.R.V. and S.F. trained

1357 the animals and collected the data. D.P., J.R.V. and W.T.N. wrote initial draft of the paper. S.I.R,

1358 D.P. and R.K. performed the surgical procedures. D.P., J.C.K., P.N., C.C. and J.B. implemented

1359 the real-time decoding setup. D.P., R.K. and C.C. designed, and D.P. and J.R.V implemented, the

1360 decoder training algorithm to obtain the decoder weights and normalization matrices. D.P. and

1361 J.R.V. analysed the data. All authors contributed analytical insights and commented on statistical

1362 tests, discussed the results and implications, and contributed extensively to the multiple subsequent

1363 drafts of the paper. 


\section{Author Information}

1367 The authors declare no competing interests. Correspondence and requests for materials should be 1368 addressed to D.P. (dpeixoto@stanford.edu), J.R.V. (jverhein@stanford.edu), or W.T.N. 1369 (bnewsome@stanford.edu).

1370 